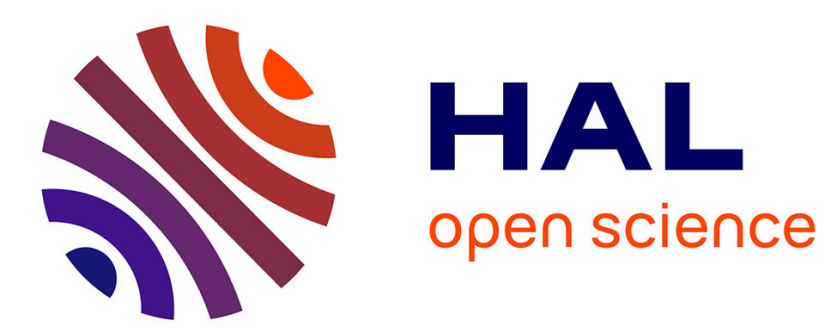

\title{
An unbiased Nitsche's formulation of large deformation frictional contact and self-contact
}

Rabii Mlika, Yves Renard, Franz Chouly

\section{To cite this version:}

Rabii Mlika, Yves Renard, Franz Chouly. An unbiased Nitsche's formulation of large deformation frictional contact and self-contact. Computer Methods in Applied Mechanics and Engineering, 2017, 325, pp.265-288. 10.1016/j.cma.2017.07.015 . hal-01427872v2

\section{HAL Id: hal-01427872 \\ https://hal.science/hal-01427872v2}

Submitted on 21 Sep 2017

HAL is a multi-disciplinary open access archive for the deposit and dissemination of scientific research documents, whether they are published or not. The documents may come from teaching and research institutions in France or abroad, or from public or private research centers.
L'archive ouverte pluridisciplinaire HAL, est destinée au dépôt et à la diffusion de documents scientifiques de niveau recherche, publiés ou non, émanant des établissements d'enseignement et de recherche français ou étrangers, des laboratoires publics ou privés. 


\title{
An unbiased Nitsche's formulation of large deformation frictional contact and self-contact
}

\author{
Rabii MLIKA ${ }^{\mathrm{a}, *}$, Yves RENARD ${ }^{\mathrm{a}, \mathrm{b}}$, Franz CHOULY $^{\mathrm{c}}$ \\ ${ }^{a}$ Université de Lyon, CNRS, INSA-Lyon, LaMCoS UMR5259, F-69621, Villeurbanne, France. \\ ${ }^{b}$ Université de Lyon, CNRS, INSA-Lyon, ICJ UMR5208, LaMCoS UMR5259, F-69621, Villeurbanne, France. \\ ${ }^{c}$ Laboratoire de Mathématiques de Besançon - UMR CNRS 6623, Université Bourgogne Franche-Comté, 16 route de Gray, 25030 \\ Besançon Cedex, France.
}

\begin{abstract}
In this paper we propose an extension of Nitsche's method for frictional contact in large elastic deformations. In fact we develop an unbiased strategy in which no asymmetry is considered between contact surfaces, conversely to the master-slave strategy. This enables to take into account within the same formalism contact of two elastic bodies as well as self-contact. We provide a numerical study of the performance and robustness of the method.
\end{abstract}

Keywords: large deformation contact, frictional contact and self-contact, Nitsche's method, unbiased formulation.

\section{Introduction}

Frictional contact problems involve difficulties from both theoretical and numerical viewpoints, especially in large deformations, where complex geometrical and mechanical quantities depend on an a priori unknown mapping between contact surfaces. Contact problems are inherently non-linear, even non-smooth, and involve variational inequalities and constrained minimization. In the literature, many attempts have been developed to deal with such problems using the finite element method. In most cases, the difficulty caused by the nondifferentiability of contact and friction laws is resolved with either a method of regularization, such as penalization or augmented Lagrangian [21, 34, or a mixed method [20, 3].

Moreover, the spatial discretization of the problem produces difficulties at level of the calculation of mechanical contact. Evaluating the quantities involved in the equations of mechanical contact is difficult when the two boundaries are discretized. The most commonly used method is the node-to-surface (NTS) approach under a master-slave configuration 24, 30. The use of the NTS method involves a loss of accuracy in the calculation of displacements and stresses in the contact area. This results from the amplification of spatial discretization errors caused by the node-wise contact constraint enforcement. A way to overcome this problem is the use of the mortar method which has been successfully applied to solve contact problems with finite deformations [15, 31, 28, In this method, the enforcement of contact constraints is applied in a weak sense throughout the contact interface. The calculation of contact can also be done by other ways such as contact domain methods [27, 17] and intermediate mortar surface method 25. A theoretical and algorithmic background for the contact between two deformable bodies undergoing large deformations is detailed in, e.g., [23, 37.

In this paper we introduce a Nitsche's method for the large deformation contact problem. Nitsche's method was originally proposed in 26 to take into account a Dirichlet condition weakly. It was adapted to bilateral contact in [18, 38 and to unilateral contact in 8, 9. This method aims at treating the interface conditions in a weak sense, thanks to a consistent penalty term. So it differs from standard penalization techniques which are nonconsistent. Conversely to mixed method and augmented Lagrangian method, the proposed approach is primal; this allows us to eliminate an outer augmentation loop as well as additional unknowns (Lagrange multiplier) and

\footnotetext{
*Corresponding author

Email addresses: rabii.mlika@insa-lyon.fr (Rabii MLIKA), yves.renard@insa-lyon.fr (Yves RENARD), franz.chouly@univ-fcomte.fr (Franz CHOULY)
} 
there is no inf-sup condition to satisfy. In [8, 9] a complete study of Nitsche's method for frictionless unilateral contact undergoing small deformations is presented. The well-posedness as well as the nominal convergence for the $H^{1}$-norm were proved. In 2 a Nitsche stabilized approach was introduced for frictional sliding problem. In [9] were introduced some variants of the method, that a real parameter $\theta$ allows to encompass. Namely, $\theta=0$ yields a non-symmetric simple version of Nitsche; when $\theta=1$ we recover a symmetric method and to $\theta=-1$ corresponds a skew-symmetric method that is much more robust regarding Nitsche's parameter. In this adaptation to the large strain case, we use a similar parameter to recover all the different variants. The above remarks concerning this parameter $\theta$ remain true to some extent for large deformation contact. The main difference between large and small strain is that, when $\theta \neq 0$, the weak formulation and the tangent problem are more difficult to obtain since they involve additional derivatives of the stress tensor.

The standard paradigm to treat the problem of two deformable bodies in contact is known as the master/slave formulation (see, e.g., 23, 24, 37]): one distinguishes between a master surface and a slave one on which one prescribes the non-penetration condition. With this paradigm important difficulties appear in the case of selfcontact and multi-body contact where it is impossible or impractical to a priori nominate a master surface and a slave one. Automating the detection and the separation between slave and master surfaces in these cases may generate a lack of robustness since it may create detection problems. To avoid these difficulties some unbiased formulations for contact were proposed, see for instance [33]. For Nitsche's method, an unbiased version for contact and Tresca friction in the small strain framework was presented in 10. In this previous work, the two contact surfaces were treated symmetrically and the integration of contact/friction condition was made along the two surfaces. This current study extends this unbiased formulation to large deformation contact and self-contact with Coulomb friction.

The reformulation of Coulomb friction follows the same path as in [7, 10. Of course no proof of well-posedness or convergence can be obtained with standard techniques for the problem under consideration, but we test numerically the performance of our method in various situations. The formulation involves boundary integrals of fields that are discretized over two different meshes what generates a difficulty for the numerical quadrature. Typically, the approach that is used in mortar-type methods is to compute elements intersections between the two contact surfaces and subdivide the integration scheme in order to accurately integrate the contributions to the algebraic system using standard Gauss quadrature techniques. This segmentation process is challenging, especially in the three dimensional case (see [31, 30]). Due to this computational complexity, it has been seen appealing to use a higher order quadrature scheme on the slave mesh without segmentation (see [14, 36]). Folowing [13, we call this technique "element-based integration" and the segmentation technique "segmentbased integration". In section 3.1 we compare the two methods: the error generated by using element-based integration, though much larger than with segment-based integration, remains small. This is in agreement with the study [13] that concludes that both methods provide acceptable results. Though segment-based integration leads to an improved quality of the solution for quadratic elements and/or friction (see [13]), we carried out the remaining part of the study with element-based integration because this method is simpler and cheaper.

Another major difficulty for large deformation contact comes from the mapping function relating the two contact surfaces. Classically, a point of the first contact surface is mapped to the closest projection point on the second one. Hence, the second surface normals $n_{y}$ govern the definition of the gap function and its kinematics. This classical mapping will in the following be simply referred to as the projection strategy. In [29] this strategy is compared with another one named ray-tracing, where a point of the first surface is mapped to the closest intersection with the other surface along the first surface normal. Unlike projection the definition of the gap and related quantities are governed by the first surface normal $n_{x}$. The formulations presented for instance in [15, 22. employ the classic projection approach, while [31, 36] present formulations that rely on the ray-tracing strategy. According to [29] the ray-tracing is more stable since the expression of the directional derivative of the mapping is quite simpler and expected to be smoother. Moreover, there are generally less special cases to treat when dealing with ray-tracing rather than projection since the probability to come across a non regular point, like a corner of the geometry or simply an element boundary, is negligible for the ray-tracing strategy while it is very frequent for the projection. In the present paper, we formulate the method for both strategies and we provide a numerical comparison between them.

In Section 1 we present the setting and notations for the problem under consideration: large deformation contact with Coulomb friction and possibly self-contact. In Section 2 the Nitsche-based approximation is detailed, particularly the variational formulation and the tangent problem. Section 3 is a numerical validation of the 
method with several tests. The influence of Nitsche's parameter for different variants is investigated numerically.

\section{Problem setting}

\subsection{Notations}

Let $\Omega \subset \mathbb{R}^{d}$ be an open bounded set that denotes the reference configuration of one or several deformable solids in a space of dimension $d=2$ or 3 . A deformed configuration $\Omega^{t}$ of the considered solids can be defined through a transformation $\varphi$ which maps any point $X$ of the reference configuration to a point $x$ of the deformed one (see Figure 1):

$$
\begin{aligned}
\varphi: \bar{\Omega} & \longrightarrow \mathbb{R}^{d} \\
X & \longmapsto x=\varphi(X) .
\end{aligned}
$$

We define the displacement $u$ relatively to the reference configuration as:

$$
u(X)=\varphi(X)-X .
$$

Deformation of the solid can be considered either in equilibrium or as part of a quasi-static evolution. To deal with Coulomb friction, a quasi-static process will be considered, and the static case can be viewed as a particular case.

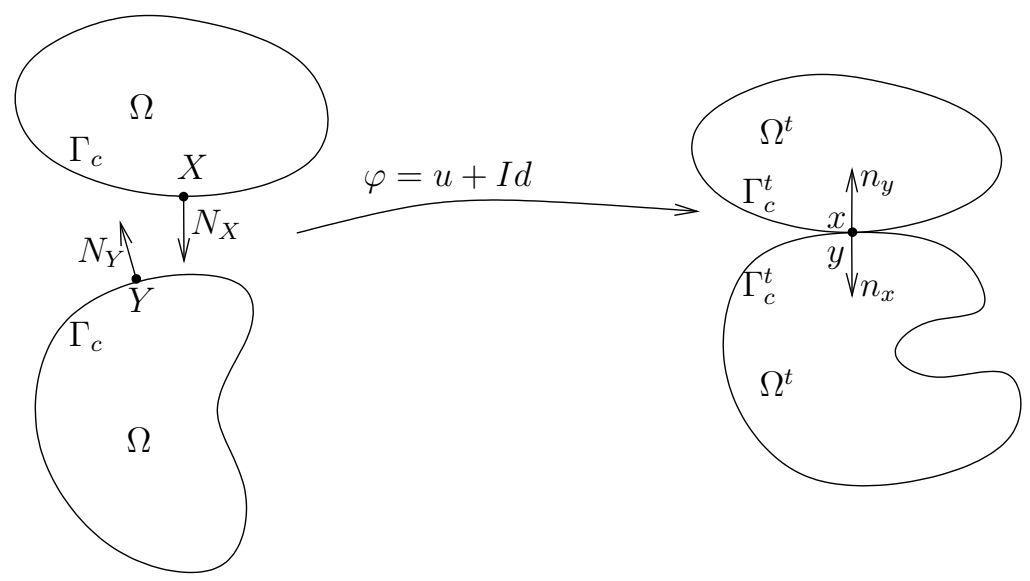

Figure 1: Basic notations for different quantities in reference and deformed configurations.

In the deformed configuration $\Omega^{t}$, at time $t$, different portions of the boundary $\partial \Omega$ of $\Omega$ may come into contact and interact with each other. In order to express this interaction mathematically, it is convenient to consider a restricted part of $\partial \Omega$ as the contact surface $\Gamma$, i.e. the surface where contact/friction phenomena may occur. As in 10 we consider an unbiased formulation that does not distinguish between a master and a slave surface. The case of self-contact is treated implicitly and there is no need to divide the self-contact surface. A non-penetration condition on the deformed contact surface $\Gamma^{t}$ can be expressed with the help of a mapping function relating a point $x$ to its mapping $y$. We denote by $\Gamma_{c}^{t} \subset \Gamma^{t}$ (resp. $\Gamma_{c} \subset \Gamma$ ) the set of points $x$ (resp. $X$ ) in the deformed (resp. reference) configuration, for which such a mapping $\Pi$ exists:

$$
\begin{aligned}
\Pi: \Gamma_{c}^{t} & \longrightarrow \Gamma^{t} \\
x & \longmapsto y=\Pi(x) .
\end{aligned}
$$

Recall that surface points $X, Y, x$ and $y$ are of dimension $d$ as well as the corresponding unit outward normal vectors: $N_{X}, N_{Y}$ in the reference configuration and $n_{x}, n_{y}$ in the deformed one.

The gradient of a quantity in the deformed (resp. reference) configuration will be noted $\nabla$ (resp. $\nabla_{X}$ ). To describe the deformation we introduce as usual the identity matrix of size $d \times d$, denoted by $\mathbf{I}$ and the deformation gradient $\mathbf{F}=\mathbf{I}+\nabla_{X} u$. We introduce as well

$$
\boldsymbol{\epsilon}(u)=\frac{1}{2}\left(\nabla u+\nabla u^{T}\right)=\frac{1}{2}\left(\left(\nabla_{X} u\right) \mathbf{F}^{-1}+\mathbf{F}^{-T}\left(\nabla_{X} u\right)^{T}\right) .
$$


The jacobian of $\varphi$ is denoted by $J=\operatorname{det} \mathbf{F}$. We introduce also the Cauchy-Green tensor $\mathbf{C}=\mathbf{F}^{T} \mathbf{F}$, and the Green-Lagrange tensor $\mathbf{E}=\frac{1}{2}(\mathbf{C}-\mathbf{I})$. We will note $\boldsymbol{\sigma}$ the Cauchy stress tensor, $\hat{\boldsymbol{\sigma}}=J \boldsymbol{\sigma} \mathbf{F}^{-T}$ the first PiolaKirchhoff stress tensor and $\mathbf{S}=J \mathbf{F}^{-1} \boldsymbol{\sigma} \mathbf{F}^{-T}$ the second Piola-Kirchhoff stress tensor. We define $\hat{\sigma}_{N}=\hat{\boldsymbol{\sigma}} N$ the contact stress on $\partial \Omega$ (where $N$ is the outward unit normal to $\partial \Omega$ ). To fix ideas we will consider a general hyperelastic constitutive law, derived from a potential $W$ that depends on the deformation through $\mathbf{E}$ (or $\mathbf{C}$ ) (see, e.g., 4, 16]), so that the second Piola-Kirchhoff stress is

$$
\mathbf{S}=\frac{\partial W}{\partial \mathbf{E}}(\mathbf{E})=2 \frac{\partial W}{\partial \mathbf{C}}(\mathbf{C})
$$

with corresponding fourth-order elasticity tensor

$$
\mathcal{C}=\frac{\partial \mathbf{S}}{\partial \mathbf{E}}=\frac{\partial^{2} W}{\partial \mathbf{E} \partial \mathbf{E}}
$$

We will need as well the isotropic tensor

$$
\mathcal{I}=\frac{1}{2}\left(\mathbf{e}_{i} \otimes \mathbf{e}_{j} \otimes \mathbf{e}_{i} \otimes \mathbf{e}_{j}+\mathbf{e}_{i} \otimes \mathbf{e}_{j} \otimes \mathbf{e}_{j} \otimes \mathbf{e}_{i}\right),
$$

where $\otimes$ denotes the tensor product of two vectors, $\left(\mathbf{e}_{i}\right)_{i=1, \ldots, d}$ is the canonical basis of $\mathbb{R}^{d}$ and where Einstein's summation convention is used. The tensor $\mathcal{I}$ has the property $\mathcal{I}: \mathbf{A}=\mathbf{A}$ for any symmetric second-order tensor A (: denotes the double-dot product between two tensors). The operator $\mathbf{T}_{n}$ refers to the projection on the tangent plane corresponding to normal vector $n$.

Since the choice of a constitutive law is not central in the description of the proposed contact approximation, we will simply denote the global potential energy by $\mathcal{J}(\cdot)$. For example, if considering simple equilibrium under a gravity force, the potential energy is

$$
\mathcal{J}(u)=\int_{\Omega} W(\mathbf{E}) d X-\int_{\Omega} \rho g \cdot u d X
$$

where $\rho$ is the density in the reference configuration and $g$ is the gravity acceleration vector. Of course, additional terms such as boundary loads, can be considered as well. Dirichlet conditions can also be prescribed, but, to simplify the formulation, the treatment of Dirichlet conditions will be omitted in the following.

Moreover, we consider the following notations to simplify the mathematical presentation. The directional derivative of a quantity $A$ with respect to the displacement $u$ in direction $\delta u$ will be denoted by $\mathcal{D} A(u)[\delta u]$ or even by $\mathcal{D} A[\delta u]$ if the argument of the quantity $A$ is not ambiguous. This directional derivative is defined as

$$
\mathcal{D} A(u)[\delta u]=\lim _{\varepsilon \rightarrow 0} \frac{A(u+\varepsilon \delta u)-A(u)}{\varepsilon}
$$

when this limit exists. The projection onto $\mathbb{R}^{-}$is defined as:

$$
[a]_{\mathbb{R}^{-}}= \begin{cases}a & \text { if } a \leq 0, \\ 0 & \text { if } a>0,\end{cases}
$$

and the projection onto a ball centered at the origin and with a radius $\tau$ is:

$$
P_{B(\tau)}(q)= \begin{cases}q & \text { if }\|q\| \leq \tau, \\ \tau \frac{q}{\|q\|} & \text { otherwise }\end{cases}
$$

where $\|\cdot\|$ is the euclidean norm on $\mathbb{R}^{d}$.

The notation $H(\cdot)$ stands for the (multivalued) Heaviside function: for any $x \in \mathbb{R}$,

$$
H(x)=\left\{\begin{array}{cc}
1 & \text { if } x>0 \\
{[0,1]} & \text { if } x=0 \\
0 & \text { if } x<0
\end{array}\right.
$$




\subsection{The mapping and the gap function}

In the problem setting above, it is assumed that a point $x$ of the deformed contact surface is mapped to a point $y$. Regarding this mapping, there are several possibilities. The most classic strategy is to define $y$ as the closest point projection of $x$ onto the deformed surface $\Gamma_{c}$, like shown in Figure 2(a). We can refer to [23] for this mapping. The main difficulty for using projection is the complicated expression of the tangent problem, that is due to the derivative of the unit normal vector $n_{y}$. The expression of this derivative can be found in [23, chapter 4]. However, this expression does not take into account the inter-element jumps.

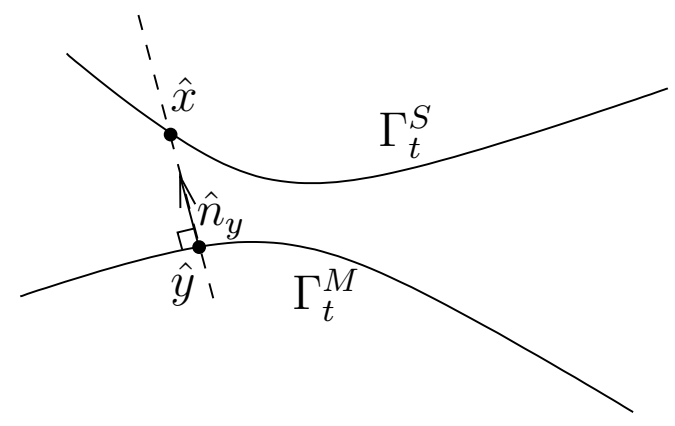

(a) Projection strategy

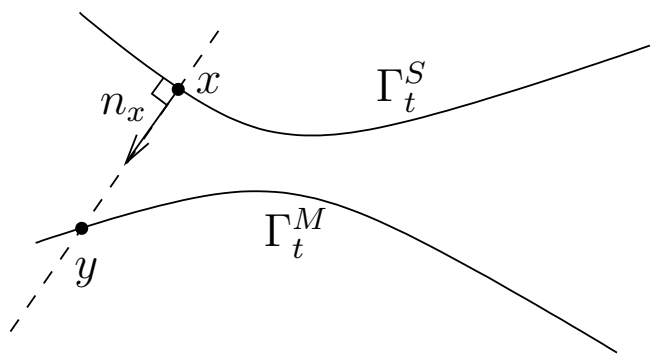

(b) Ray-tracing strategy

Figure 2: Illustration of projection and ray-tracing strategies.

An alternative strategy, corresponding to Fig. 2(b), is to define $y$ as the closest intersection of the contact surface with the line passing through point $x$ and having direction vector $n_{x}$. The latter strategy, which can be referred to as ray-tracing, was studied in 29. Gap functions corresponding to ray-tracing and projection with respect to a point $x(X)$ are respectively defined by:

$$
\begin{aligned}
& g=n_{x} \cdot(y-x), \quad \text { for ray-tracing } \\
& \text { and } g=n_{y} \cdot(x-y), \quad \text { for projection, }
\end{aligned}
$$

To obtain and to linearize a weak formulation associated to the non-penetration condition, we need the directional derivatives of all quantities involved in (1) (resp. (2)) with respect to the displacement $u$ in a virtual direction $\delta u$ (see [29, 23, 22]) for detailed expressions.

\subsection{Formulation of contact and friction conditions}

The impenetrability constraint is stated mathematically as $g(u) \geq 0$. To formulate the associated complementarity conditions, we need to consider the contact traction $\hat{\sigma}_{N}(u)$ which we take to be the Piola traction at point $X$. This traction is resolved as follows:

$$
\hat{\sigma}_{N}(u)=\hat{\sigma}_{n}(u) n_{x}+\mathbf{T}_{n_{x}} \hat{\sigma}_{N}(u)=\hat{\sigma}_{n}(u) n_{x}+\hat{\sigma}_{t},
$$

where $n_{x}$ is the outward normal to $\Gamma_{c}^{t}$ at $x$. When contact occurs $\left(\hat{\sigma}_{n}<0\right)$ the outward normal vectors $n_{x}$ and $n_{y}$ are opposite. Thus, for projection the stress vector $\hat{\sigma}_{N}$ is resolved at the projected point $y$ according the normal vector $-n_{y}$, instead of $n_{x}$. The quantity $\hat{\sigma}_{n}(u)$ now represents the contact pressure at $X$, and must be negative. The conditions for normal contact are:

$$
\begin{aligned}
g(u) & \geq 0 \\
\hat{\sigma}_{n}(u) & \leq 0 \\
\hat{\sigma}_{n}(u) g(u) & =0
\end{aligned} \quad \text { on } \Gamma_{c} .
$$

Let $\gamma$ be a given positive function. As in [1, 8, 9], the contact conditions $(3 \mathrm{a})-(3 \mathrm{~b})-(3 \mathrm{c})$ are reformulated as

$$
\hat{\sigma}_{n}(u)=\left[\hat{\sigma}_{n}(u)+\gamma g(u)\right]_{\mathbb{R}^{-}} .
$$


In the presence of friction, normal and tangential stresses at the contact interface are coupled through the sliding velocity vector $\mathrm{v}$. As in [29, we use the frame indifferent definition of velocity described in [12]. Adapted to the current notation $\mathrm{v}(X)$ reads:

$$
\mathrm{v}(X)=\dot{\varphi}(X)-\dot{\varphi}(Y)+g \dot{n},
$$

with dotted quantities representing time derivatives and $n$ refers to $n_{x}$ for ray-tracing and $-n_{y}$ for projection. Time discretization is based on a backward Euler approximation of the first expression in (5) which reads:

$$
\mathrm{v}(X)=\frac{1}{\Delta t}(\varphi(X)-\varphi(Y)+g n)-\frac{1}{\Delta t}\left(\varphi_{0}(X)-\varphi_{0}(Y)+g n_{0}\right),
$$

where $\Delta t>0$ is the time-step, and where $\varphi_{0}, n_{0}$ are respectively the deformation and the surface normal at the previous time-step. Equation (6) can be then simplified, as in [29, using (1) and (2):

$$
\mathrm{v}(X)=-\frac{1}{\Delta t}\left(\varphi_{0}(X)-\varphi_{0}(Y)+g n_{0}\right) .
$$

It should be underlined here that the mapping between points $X$ and $Y$ appearing in (7) corresponds to the current deformation $\varphi$ and not to the deformation $\varphi_{0}$ at previous time-step.

The conditions of Coulomb friction can be written as follows:

$$
\begin{cases}\left\|\hat{\sigma}_{t}(u)\right\| \leq-\mathscr{F} \hat{\sigma}_{n}(u) & \text { if } \mathrm{v}=0, \\ \hat{\sigma}_{t}(u)=\mathscr{F} \hat{\sigma}_{n}(u) \frac{\mathrm{v}}{\|\mathrm{v}\|} & \text { otherwise. }\end{cases}
$$

As presented in [7 for Tresca friction, we could reformulate the Coulomb friction condition using the projection $P_{B(\tau)}$. In fact, for a given positive function $\gamma$, the friction condition is equivalent to the non-smooth equation:

$$
\hat{\sigma}_{t}(u)=P_{B\left(-\mathscr{F} \hat{\sigma}_{n}(u)\right)}\left(\hat{\sigma}_{t}(u)-\gamma \mathrm{v}\right) .
$$

To simplify the formulation, and following [29], we define the non-smooth operator $\mathcal{C}_{\gamma, \mathscr{F}}$ as:

$$
\mathcal{C}_{\gamma, \mathscr{F}}(\sigma, g, \mathrm{v}, n)=[\sigma \cdot n+\gamma g]_{\mathbb{R}^{-}} n+\mathcal{P}_{B\left(n,-\mathscr{F}[\sigma \cdot n+\gamma g]_{\mathbb{R}^{-}}\right.}(\sigma-\gamma \mathrm{v}) .
$$

Unlike $P_{B(\tau)}$ that represents a simple ball projection, here $\mathcal{P}_{B(n, \tau)}$ is the projection onto the tangent plane defined by the normal $n$, followed by the projection onto a ball of radius $\tau$, i.e. :

$$
\mathcal{P}_{B(n, \tau)}(q)= \begin{cases}\mathbf{T}_{n} q & \text { if }\left\|\mathbf{T}_{n} q\right\| \leq \tau, \\ \tau \frac{\mathbf{T}_{n} q}{\left\|\mathbf{T}_{n} q\right\|} & \text { otherwise. }\end{cases}
$$

As a result, contact and friction conditions, in the case of ray-tracing, are formulated as:

$$
\hat{\sigma}_{N}=\mathcal{C}_{\gamma, \mathscr{F}}\left(\hat{\sigma}_{N}, g, \mathrm{v}, n_{x}\right) .
$$

\section{A Nitsche-based formulation for frictional contact}

\subsection{Weak formulation}

We consider test functions $\delta u \in V_{0}$, with $V_{0}$ the space of all (smooth) admissible variations of $u$ satisfying possibly homogeneous Dirichlet conditions on the appropriate part of $\partial \Omega$. The abstract weak formulation for (frictional) contact is:

$$
G(u ; \delta u)=\int_{\Gamma_{c}} \hat{\sigma}_{N} \cdot \delta u d \Gamma, \quad \forall \delta u \in V_{0} .
$$

The expression $G(u ; \delta u)$ is the sum of the internal virtual work and of the virtual work of body or surface external forces. This work is seen to balance the virtual work of the contact and friction forces acting on 
$\Gamma_{c}$. In the considered case of hyperelastic bodies and simple equilibrium under a gravity force, there holds $G(u ; \delta u)=\mathcal{D} \mathcal{J}(u)[\delta u]$. Thus, the above weak formulation reads:

$$
\mathcal{D} \mathcal{J}(u)[\delta u]-\int_{\Gamma_{c}} \hat{\sigma}_{N} \cdot \delta u d \Gamma=0, \quad \forall \delta u \in V_{0} .
$$

Moreover, we apply the second Newton law: to each point $X \in \Gamma_{c}$, we require that the differential contact force induced on $\Gamma_{c}$ at the corresponding point $Y$ be equal and opposite to that produced at $X$, i.e.

$$
\hat{\sigma}_{N}(X) d \Gamma_{X}=-\hat{\sigma}_{N}(Y) d \Gamma_{Y} .
$$

Integrating with respect to $Y$ instead of $X$, we obtain the following identities:

$$
\begin{aligned}
\int_{\Gamma_{c}} \hat{\sigma}_{N}(X) \cdot \delta u(X) d \Gamma_{X} & =\int_{\Gamma_{c}} \hat{\sigma}_{N}(Y) \cdot \delta u(Y) d \Gamma_{Y} \\
& =\frac{1}{2}\left(\int_{\Gamma_{c}} \hat{\sigma}_{N}(X) \cdot \delta u(X) d \Gamma_{X}+\int_{\Gamma_{c}} \hat{\sigma}_{N}(Y) \cdot \delta u(Y) d \Gamma_{Y}\right) .
\end{aligned}
$$

Using 13 we get:

$$
\int_{\Gamma_{c}} \hat{\sigma}_{N}(X) \cdot \delta u(X) d \Gamma_{X}=\frac{1}{2} \int_{\Gamma_{c}} \hat{\sigma}_{N}(X) \cdot(\delta u(X)-\delta u(Y)) d \Gamma_{X} .
$$

We inject the above expression into $(12)$ and get:

$$
\mathcal{D} \mathcal{J}(u)[\delta u]-\frac{1}{2} \int_{\Gamma_{c}} \hat{\sigma}_{N} \cdot(\delta u(X)-\delta u(Y)) d \Gamma=0, \quad \forall \delta u \in V_{0} .
$$

Let now $\theta \in \mathbb{R}$ be a fixed parameter that we use to recover different variants of the Nitsche method, as in the linear elastic setting (see, e.g., 9]). With the splitting

$$
\begin{aligned}
\delta u(X)-\delta u(Y) & =-\frac{1}{\gamma}\left(\gamma(\delta u(Y)-\delta u(X))+\theta \mathcal{D} \hat{\sigma}_{N}[\delta u]\right)+\frac{\theta}{\gamma} \mathcal{D} \hat{\sigma}_{N}[\delta u] \\
& =-\frac{1}{\gamma} \mathcal{D}\left(\theta \hat{\sigma}_{N}+\gamma(u(Y)-u(X))\right)[\delta u]+\frac{\theta}{\gamma} \mathcal{D} \hat{\sigma}_{N}[\delta u]
\end{aligned}
$$

we obtain, for all $\delta u \in V_{0}$,

$$
\mathcal{D} \mathcal{J}(u)[\delta u]-\frac{1}{2} \int_{\Gamma_{c}} \frac{\theta}{\gamma} \hat{\sigma}_{N} \cdot \mathcal{D} \hat{\sigma}_{N}[\delta u] d \Gamma+\frac{1}{2} \int_{\Gamma_{c}} \frac{1}{\gamma} \hat{\sigma}_{N} \cdot \mathcal{D}\left(\theta \hat{\sigma}_{N}+\gamma(u(Y)-u(X))\right)[\delta u] d \Gamma=0 .
$$

We inject finally the expression (11) into 14 and obtain, formally, our Nitsche's based formulation for frictional contact and the ray-tracing strategy:

$$
\left\{\begin{array}{l}
\mathcal{D} \mathcal{J}(u)[\delta u]-\frac{1}{2} \int_{\Gamma_{c}} \frac{\theta}{\gamma} \hat{\sigma}_{N} \cdot \mathcal{D} \hat{\sigma}_{N}[\delta u] d \Gamma \\
+\frac{1}{2} \int_{\Gamma_{c}} \frac{1}{\gamma} \mathcal{C}_{\gamma, \mathscr{F}}\left(\hat{\sigma}_{N}, g, \mathrm{v}, n_{x}\right) \cdot \mathcal{D}\left(\theta \hat{\sigma}_{N}+\gamma(u(Y)-u(X))\right)[\delta u] d \Gamma=0 \quad \forall \delta u \in V_{0} .
\end{array}\right.
$$

The expression of $\mathcal{D} \hat{\sigma}_{N}[\delta u]$ is provided in AppendixA Note that, for the numerical solving, when $\theta \neq 0$, the tangent system involves the second order derivative: $\mathcal{D}^{2} \hat{\boldsymbol{\sigma}}(u)[\delta u, \Delta u]$ (see Section 2.3 ). This emphasizes the interest of the non-symmetric variant $\theta=0$ for which the method is simpler. As in the small strain case [9, 10 , the interest of the symmetric variant $\theta=1$ consists mostly in its derivation from a potential (see Section 2.2 ) and the symmetry of the tangent problem, while the interest of the skew-symmetric variant $\theta=-1$ is its robustness respectively to the Nitsche parameter $\gamma$ (see Section 3). 
Remark 2.1. For projection we decompose $\hat{\sigma}_{N}$ at point $y$ instead of $x$ and using the normal $n_{y}$. The yielding formulation reads:

$$
\left\{\begin{array}{l}
\mathcal{D} \mathcal{J}(u)[\delta u]-\frac{1}{2} \int_{\Gamma_{c}} \frac{\theta}{\gamma} \hat{\sigma}_{N} \cdot \mathcal{D} \hat{\sigma}_{N}[\delta u] d \Gamma \\
+\frac{1}{2} \int_{\Gamma_{c}} \frac{1}{\gamma} \mathcal{C}_{\gamma, \mathscr{F}}\left(\hat{\sigma}_{N}, g, \mathrm{v}, n_{y}\right) \cdot \mathcal{D}\left(\theta \hat{\sigma}_{N}+\gamma(u(Y)-u(X))\right)[\delta u] d \Gamma=0 \quad \forall \delta u \in V_{0} .
\end{array}\right.
$$

In this case, the derivative of the gap is (following, e.g., [23, [29]):

$$
\mathcal{D} g[\delta u]=n_{y} \cdot(\delta u(X)-\delta u(Y)) .
$$

This results allows us to get a symmetric variant of the Nitsche's formulation in the frictionless case and when $\theta=1$. This variant is similar to the formulation described in Proposition 2.3. When projecting on $n=-n_{y}$, the method reads indeed:

$$
\left\{\begin{array}{l}
\mathcal{D} \mathcal{J}(u)[\delta u]-\frac{1}{2} \int_{\Gamma_{c}} \frac{1}{\gamma} \hat{\sigma}_{n} \mathcal{D} \hat{\sigma}_{n}[\delta u] d \Gamma \\
+\frac{1}{2} \int_{\Gamma_{c}} \frac{1}{\gamma}\left[\hat{\sigma}_{n}+\gamma g\right]_{\mathbb{R}^{-}}\left(\mathcal{D} \hat{\sigma}_{n}[\delta u]+\gamma \mathcal{D} g[\delta u]\right) d \Gamma=0 \quad \forall \delta u \in V_{0} .
\end{array}\right.
$$

Remark 2.2. A biased version of our Nitsche's method is obtained by dividing the domain $\Omega$ into two bodies $\Omega^{1}$ and $\Omega^{2}$ and the contact surface $\Gamma_{c}$ into a master surface $\Gamma_{c}^{M}$ and a slave one $\Gamma_{c}^{S}$. In this case the factor $\frac{1}{2}$ disappears when applying the second Newton law because the integration is applied only on the slave surface. In this case the method reads:

$$
\left\{\begin{array}{l}
\mathcal{D} \mathcal{J}(u)[\delta u]-\int_{\Gamma_{c}^{S}} \frac{\theta}{\gamma} \hat{\sigma}_{N} \cdot \mathcal{D} \hat{\sigma}_{N}[\delta u] d \Gamma \\
+\int_{\Gamma_{c}^{S}} \frac{1}{\gamma} \mathcal{C}_{\gamma, \mathscr{F}}\left(\hat{\sigma}_{N}, g, \mathrm{v}, n_{x}\right) \cdot \mathcal{D}\left(\theta \hat{\sigma}_{N}+\gamma(u(Y)-u(X))\right)[\delta u] d \Gamma=0 \quad \forall \delta u \in V_{0} .
\end{array}\right.
$$

\subsection{Energy potential and symmetric formulation for frictionless contact}

In this section, we show, that, at least formally, a symmetric variant $\theta=1$ of Nitsche's formulation, close to 15, for frictionless contact derives from an energy potential. This result is summarized as:

Proposition 2.3. Let us define the energy potential $\mathcal{J}_{\mathrm{N}}(\cdot)$ that takes into account the body deformation as well as non-penetration formulated in a Nitsche's manner:

$$
\mathcal{J}_{\mathrm{N}}(u)=\mathcal{J}(u)-\frac{1}{4} \int_{\Gamma_{c}} \frac{1}{\gamma} \hat{\sigma}_{n}^{2} d \Gamma+\frac{1}{4} \int_{\Gamma_{c}} \frac{1}{\gamma}\left[\hat{\sigma}_{n}+\gamma g\right]_{\mathbb{R}^{-}}^{2} d \Gamma,
$$

where $\gamma>0$ is the Nitsche's parameter, $\hat{\sigma}_{n}$ is the normal stress in reference configuration (see Section 1.3) and $g$ is the gap function (see Section 1.2). The first-order optimality system associated to $\mathcal{J}_{\mathrm{N}}(\cdot)$ reads:

$$
\left\{\begin{array}{l}
\mathcal{D} \mathcal{J}(u)[\delta u]-\frac{1}{2} \int_{\Gamma_{c}} \frac{1}{\gamma} \hat{\sigma}_{n} \mathcal{D} \hat{\sigma}_{n}[\delta u] d \Gamma \\
+\frac{1}{2} \int_{\Gamma_{c}} \frac{1}{\gamma}\left[\hat{\sigma}_{n}+\gamma g\right]_{\mathbb{R}^{-}} \mathcal{D}\left(\hat{\sigma}_{n}+\gamma g\right)[\delta u] d \Gamma=0 \quad \forall \delta u \in V_{0}
\end{array}\right.
$$

Proof: Let us write the optimality system associated to $\mathcal{J}_{\mathrm{N}}(\cdot)$ :

$$
\mathcal{D} \mathcal{J}_{\mathrm{N}}(u)[\delta u]=\mathcal{D} \mathcal{J}(u)[\delta u]-\frac{1}{2} \int_{\Gamma_{c}} \frac{1}{\gamma} \hat{\sigma}_{n} \mathcal{D} \hat{\sigma}_{n}[\delta u] d \Gamma+\frac{1}{4} \int_{\Gamma_{c}} \frac{1}{\gamma} \mathcal{D}\left[\hat{\sigma}_{n}+\gamma g\right]_{\mathbb{R}^{-}}^{2}[\delta u] d \Gamma=0 \quad \forall \delta u \in V_{0} .
$$

To obtain (19) there remains to compute:

$$
\begin{aligned}
\mathcal{D}\left[\hat{\sigma}_{n}+\gamma g\right]_{\mathbb{R}^{-}}^{2}[\delta u] & =2\left[\hat{\sigma}_{n}+\gamma g\right]_{\mathbb{R}^{-}} \mathcal{D}\left[\hat{\sigma}_{n}+\gamma g\right]_{\mathbb{R}^{-}}[\delta u] \\
& =2\left[\hat{\sigma}_{n}+\gamma g\right]_{\mathbb{R}^{-}} H\left(-\left(\hat{\sigma}_{n}+\gamma g\right)\right) \mathcal{D}\left(\hat{\sigma}_{n}+\gamma g\right)[\delta u] \\
& =2\left[\hat{\sigma}_{n}+\gamma g\right]_{\mathbb{R}^{-}} \mathcal{D}\left(\hat{\sigma}_{n}+\gamma g\right)[\delta u],
\end{aligned}
$$


where we used properties $\mathcal{D}[A(u)]_{\mathbb{R}^{-}}[\delta u]=H(-A(u)) \mathcal{D} A(u)[\delta u]$, for any application $A: u \mapsto A(u) \in \mathbb{R}$ as well as $H(-x)[x]_{\mathbb{R}^{-}}=[x]_{\mathbb{R}^{-}}$for any $x \in \mathbb{R}$.

The expression of the derivative $\mathcal{D} \hat{\sigma}_{n}[\delta u]$ is detailed in AppendixA while for $\mathcal{D} g[\delta u]$, we can refer to [29, Section 3] both for ray-tracing and projection techniques.

Remark 2.4. We can introduce $\theta \in \mathbb{R}$, as in section 1.3. We only modify slightly system (19) as below:

$$
\left\{\begin{array}{l}
\mathcal{D} \mathcal{J}(u)[\delta u]-\frac{1}{2} \int_{\Gamma_{c}} \frac{\theta}{\gamma} \hat{\sigma}_{n} \mathcal{D} \hat{\sigma}_{n}[\delta u] d \Gamma \\
+\frac{1}{2} \int_{\Gamma_{c}} \frac{1}{\gamma}\left[\hat{\sigma}_{n}+\gamma g\right]_{\mathbb{R}^{-}} \mathcal{D}\left(\theta \hat{\sigma}_{n}+\gamma g\right)[\delta u] d \Gamma=0 \quad \forall \delta u .
\end{array}\right.
$$

\subsection{Finite element approximation and tangent system}

A standard Galerkin procedure can be applied by choosing a finite element space for the displacement, i.e., $V_{0}^{h} \subset V_{0}$ to account for any possible Dirichlet condition. We consider in what follows that $\gamma$ is a positive piecewise constant function on the contact interface $\Gamma_{c}$ which satisfies

$$
\gamma_{\mid K \cap \Gamma_{c}}=\frac{\gamma_{0}}{h_{K}}
$$

for every element $K$ that has a non-empty intersection of dimension $d-1$ with $\Gamma_{c}$, where $h_{K}$ is the size of the element $K$ and $\gamma_{0}$ is a positive given constant. Note that the value of $\gamma$ on element intersections has no influence.

Then, the finite element approximation of System (15) reads:

$$
\left\{\begin{array}{l}
\mathcal{D} \mathcal{J}\left(u^{h}\right)\left[\delta u^{h}\right]-\frac{1}{2} \int_{\Gamma_{c}} \frac{\theta}{\gamma} \hat{\sigma}_{N}^{h} \cdot \mathcal{D} \hat{\sigma}_{N}^{h}\left[\delta u^{h}\right] d \Gamma \\
+\frac{1}{2} \int_{\Gamma_{c}} \frac{1}{\gamma} \mathcal{C}_{\gamma, \mathscr{F}}\left(\hat{\sigma}_{N}^{h}, g, \mathrm{v}^{h}, n_{x}\right) \cdot \mathcal{D}\left(\theta \hat{\sigma}_{N}^{h}+\gamma\left(u^{h}(Y)-u^{h}(X)\right)\right)\left[\delta u^{h}\right] d \Gamma=0 \quad \forall \delta u^{h} \in V_{0}^{h},
\end{array}\right.
$$

where $\hat{\sigma}_{N}^{h}=\hat{\sigma}_{N}\left(u^{h}\right), \mathcal{D} \hat{\sigma}_{N}^{h}\left[\delta u^{h}\right]=\mathcal{D} \hat{\sigma}_{N}\left(u^{h}\right)\left[\delta u^{h}\right]$ and $\mathrm{v}^{h}$ is a finite element approximation of the velocity v. The system 21] is Lipschitz-continuous with respect to $u^{h}$ and piecewise $C^{1}$-continuous. This means that it is sufficiently regular to be solved with a generalized Newton method. The tangent system is provided below. Each Newton step consists in finding $\Delta u^{h}$ solution to:

$$
\left\{\begin{array}{l}
\mathcal{D}^{2} \mathcal{J}\left(u^{h}\right)\left[\delta u^{h}, \Delta u^{h}\right] \\
-\frac{1}{2} \int_{\Gamma_{c}} \frac{\theta}{\gamma} \mathcal{D} \hat{\sigma}_{N}^{h}\left[\Delta u^{h}\right] \cdot \mathcal{D} \hat{\sigma}_{N}^{h}\left[\delta u^{h}\right] d \Gamma-\frac{1}{2} \int_{\Gamma_{c}} \frac{\theta}{\gamma} \hat{\sigma}_{N}^{h} \cdot \mathcal{D}^{2} \hat{\sigma}_{N}^{h}\left[\delta u^{h}, \Delta u^{h}\right] d \Gamma \\
+\frac{1}{2} \int_{\Gamma_{c}} \frac{1}{\gamma}\left(\partial_{\sigma} \mathcal{C}_{\gamma, \mathscr{F}} \mathcal{D} \hat{\sigma}_{N}^{h}\left[\Delta u^{h}\right]+\partial_{g} \mathcal{C}_{\gamma, \mathscr{F}} \mathcal{D} g\left[\Delta u^{h}\right]+\partial_{\mathrm{v}} \mathcal{C}_{\gamma, \mathscr{F}} \mathcal{D v}^{h}\left[\Delta u^{h}\right]+\partial_{n} \mathcal{C}_{\gamma, \mathscr{F}} \mathcal{D} n_{x}\left[\Delta u^{h}\right]\right) \\
\quad \cdot \mathcal{D}\left(\theta \hat{\sigma}_{N}^{h}+\gamma\left(u^{h}(Y)-u^{h}(X)\right)\right)\left[\delta u^{h}\right] d \Gamma \\
+\frac{1}{2} \int_{\Gamma_{c}} \frac{1}{\gamma} \mathcal{C}_{\gamma, \mathscr{F}}\left(\hat{\sigma}_{N}^{h}, g, \mathrm{v}^{h}, n_{x}\right) \cdot\left(\theta \mathcal{D}^{2} \hat{\sigma}_{N}^{h}\left[\delta u^{h}, \Delta u^{h}\right]+\gamma \nabla_{X} \delta u^{h}(Y) \mathcal{D} Y\left[\Delta u^{h}\right]\right) d \Gamma \\
=-\mathcal{D} \mathcal{J}\left(u^{h}\right)\left[\delta u^{h}\right]+\frac{1}{2} \int_{\Gamma_{c}} \frac{\theta}{\gamma} \hat{\sigma}_{N}^{h} \cdot \mathcal{D} \hat{\sigma}_{N}^{h}\left[\delta u^{h}\right] d \Gamma \\
-\frac{1}{2} \int_{\Gamma_{c}} \frac{1}{\gamma} \mathcal{C}_{\gamma, \mathscr{F}}\left(\hat{\sigma}_{N}^{h}, g, \mathrm{v}^{h}, n_{x}\right) \cdot \mathcal{D}\left(\theta \hat{\sigma}_{N}^{h}+\gamma\left(u^{h}(Y)-u^{h}(X)\right)\right)\left[\delta u^{h}\right] d \Gamma \quad \forall \delta u^{h} \in V_{0}^{h},
\end{array}\right.
$$

where $\mathcal{D}^{2} \mathcal{J}\left(u^{h}\right)\left[\delta u^{h}, \Delta u^{h}\right]$ is the second directional derivative of $\mathcal{J}\left(u^{h}\right)$. From 77 the derivative $\mathcal{D} \mathrm{v}^{\mathrm{h}}\left[\Delta u^{h}\right]$ can be evaluated as:

$$
\mathcal{D} \mathrm{v}^{\mathrm{h}}\left[\Delta u^{h}\right]=\frac{1}{\Delta t}\left(\mathbf{F}_{Y 0} \mathcal{D} Y\left[\Delta u^{h}\right]-\mathcal{D} g\left[\Delta u^{h}\right] n_{0}\right),
$$

where $\mathbf{F}_{Y 0}$ is the deformation gradient at the previous time-step, evaluated at point $Y$. All partial derivatives $\partial_{\sigma} \mathcal{C}, \partial_{g} \mathcal{C}, \partial_{\mathrm{v}} \mathcal{C}$ and $\partial_{n} \mathcal{C}$ of the function $\mathcal{C}$ are provided in 29, Appendix A] with small changes of notations since the negative part $[x]_{-}\left(=-[x]_{\mathbb{R}^{-}}\right)$is used in $[29]$ instead of the projection onto $\mathbb{R}^{-}$. 
For the ray-tracing strategy, we refer to [29] for the exact expression of $\mathcal{D} g\left[\Delta u^{h}\right], \mathcal{D} n_{x}\left[\Delta u^{h}\right]$ and and $\mathcal{D} Y\left[\Delta u^{h}\right]$. For the projection strategy, the tangent system is the same, replacing $n_{x}$ by $n_{y}$. In this case $\mathcal{D} g\left[\Delta u^{h}\right]$ is simple to compute but the expression of $\mathcal{D} n_{y}\left[\Delta u^{h}\right]$ is quite intricated. Therefore we neglect this term in the tangent system and in the expression of $\mathcal{D} Y\left[\Delta u^{h}\right]$. Using $[2] \mathcal{D} y[\delta u]$ reads:

$$
\mathcal{D} y[\delta u]=\delta u(X)-\mathcal{D} g[\delta u] n_{y}-g \mathcal{D} n_{y}[\delta u] \simeq \delta u(X)-\mathcal{D} g[\delta u] n_{y} .
$$

Moreover there also holds (see, e.g., 29])

$$
\mathcal{D} y[\delta u]=\delta u(Y)+\mathbf{F}_{Y} \mathcal{D} Y[\delta u], \quad \mathcal{D} g[\delta u]=n_{y} \cdot(\delta u(X)-\delta u(Y)) .
$$

Finally, for the projection strategy, when neglecting $\mathcal{D} n_{y}[\delta u]$, we can take:

$$
\mathcal{D} Y[\delta u] \simeq \mathbf{F}_{Y}^{-1}\left(\mathbf{I}-n_{y} \otimes n_{y}\right)(\delta u(X)-\delta u(Y)) .
$$

We note that for projection, even when calculating the exact expression of the derivatives, the discrete method will not be stable because of the non-continuity of the normal vector $n_{y}(X)$ in terms of the position $X$ since the projection is not a continuous operator.

Remark 2.5. For the non-symmetric variant $\theta=0$, the tangent system can be substantially simplified as:

$$
\left\{\begin{array}{c}
\mathcal{D}^{2} \mathcal{J}\left(u^{h}\right)\left[\delta u^{h}, \Delta u^{h}\right] \\
+\frac{1}{2} \int_{\Gamma_{c}}\left(\partial_{\sigma} \mathcal{C}_{\gamma, \mathscr{F}} \mathcal{D} \hat{\sigma}_{N}^{h}\left[\Delta u^{h}\right]+\partial_{g} \mathcal{C}_{\gamma, \mathscr{F}} \mathcal{D} g\left[\Delta u^{h}\right]+\partial_{\mathrm{v}} \mathcal{C}_{\gamma, \mathscr{F}} \mathcal{D v}^{h}\left[\Delta u^{h}\right]+\partial_{n} \mathcal{C}_{\gamma, \mathscr{F}} \mathcal{D} n_{x}\left[\Delta u^{h}\right]\right) \\
\cdot\left(\delta u^{h}(Y)-\delta u^{h}(X)\right) d \Gamma+\frac{1}{2} \int_{\Gamma_{c}} \mathcal{C}_{\gamma, \mathscr{F}}\left(\hat{\sigma}_{N}^{h}, g, \mathrm{v}^{h}, n_{x}\right) \cdot\left(\nabla_{X} \delta u^{h}(Y) \mathcal{D} Y\left[\Delta u^{h}\right]\right) d \Gamma \\
\left.=-\mathcal{D} \mathcal{J}\left(u^{h}\right)\left[\delta u^{h}\right]-\frac{1}{2} \int_{\Gamma_{c}} \mathcal{C}_{\gamma, \mathscr{F}}\left(\hat{\sigma}_{N}^{h}, g, \mathrm{v}^{h}, n_{x}\right) \cdot\left(\delta u^{h}(Y)-\delta u^{h}(X)\right)\right) d \Gamma \quad \forall \delta u^{h} \in V_{0}^{h} .
\end{array}\right.
$$

\section{Numerical tests and validation}

Formulation (21) has been implemented in our open-source finite element library GetFEM++ (see http: //getfem.org/). It corresponds to solving System 22 within a (semi-smooth) Newton loop. We test and compare both unbiased and biased versions, as well as variants corresponding to different values of $\theta=-1,0,1$. Except in Section 3.6, the mapping strategy used is always the ray-tracing.

As a first example, the simulation of a two-dimensional patch test with non-matching meshes allows to check the capability of the formulation to exactly transmit constant normal stresses between two contacting surfaces, regardless of their discretization. With this first example, we provide numerical evidence that element-based integration leads to acceptable accuracy, provided enough Gauss points are used. So we keep this choice for the remaining numerical tests, following [14] and [29]. The second test is the two-dimensional Hertz contact problem that assesses the capability of the approximation to capture a known contact pressure profile in a restricted contact area with non-matching meshes. Further two-dimensional examples are classic problems found in the large sliding contact literature and aim at testing the performance of the proposed method. Finally, simulation of contact between two hollow cylindrical tubes, including self-contact, is presented in order to evaluate the performance of the method in three dimensions and for the self-contact case. In reference [29] the projection and the ray-tracing strategies are presented with a general discussion. We complement here this discussion with a numerical comparaison between the two mapping strategies.

\subsection{Taylor patch test}

The patch test originally proposed in [35, investigates the ability of contact formulations to exactly transmit constant normal tractions between two contacting surfaces, regardless of their discretization. However, note that a patch test does not provide any information about the stability of an algorithm and and is not relevant when considering contact between deformable and rigid bodies. In this section we compare the obtained error with element-based and segment-based quadrature. Among available patch tests, we choose the one depicted in Figure 3. that is similar to the test used in [11] and [19]. An elastic body rests on a smooth elastic foundation having 


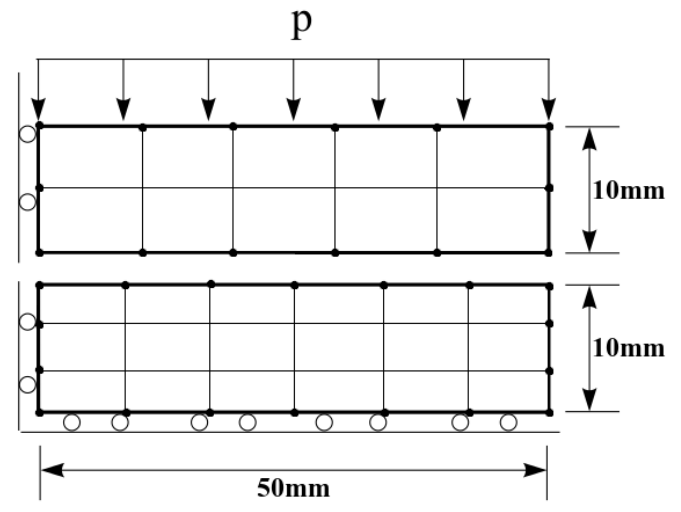

Figure 3: Taylor patch test configuration.

the same dimensions. A uniform distributed load $p=10 \mathrm{KPa}$ is applied on the upper surface of the solid. A two dimensional plain strain analysis is considered with a Saint Venant-Kirchhoff material. Corresponding elastic parameters are: $E=2 \cdot 10^{5} \mathrm{MPa}$ and $\nu=0.3$. Frictionless contact is considered.

We compare the performance of our Nitsche-based method with some other discretizations of contact. The transferred contact stress is measured on the lower contact surface. We compare the obtained pressure profile with those computed using a simple nodal (node-to-segment) approximation, with an integral augmented lagrangian method (see [29]) as well as a mortar method (see [36, 31]). For Nitsche's method, we compare biased and unbiased versions. For the biased method, as well as for other contact methods, the slave surface is the upper one. The parameters for Nitsche's method are: $\theta=0$ and $\gamma_{0}=100 E$. At first, we perform the test with a subdivsion of the quadrature method according to the elements intersections (segment-based integration). The maximum relative stress errors for different methods is shown in Table 1 with 4 integration points per element and Lagrange $\mathbb{Q}_{2}$ elements. All of Nitsche's methods, augmented Lagrangian and mortar ones produce a resulting axial stress exactly satisfied to more than ten decimal places and a zero penetration up to the truncation error. As expected the nodal method results in a much higher error.

\begin{tabular}{cccccc}
\hline Approximation method & Biased Nitsche & Unbiased Nitsche & Augmented Lag & Mortar & Nodal \\
\hline \hline Max pressure relative error & $7.5 \cdot 10^{-12}$ & $3.7 \cdot 10^{-12}$ & $8.8 \cdot 10^{-9}$ & $1.7 \cdot 10^{-13}$ & $1.1 \cdot 10^{-1}$ \\
\hline Max gap $(\mathrm{mm})$ & $-8.5 \cdot 10^{-16}$ & $-6.4 \cdot 10^{-16}$ & $-2.5 \cdot 10^{-12}$ & $-6.5 \cdot 10^{-19}$ & $-9 \cdot 10^{-6}$ \\
\hline \hline
\end{tabular}

Table 1: Maximum of contact pressure relative error and gap for segment-based integration.

If, now, performing same measures using the Gauss integration scheme without cutting (element-base integration) we get the following results:

\begin{tabular}{cccccc}
\hline Approximation method & Biased Nitsche & Unbiased Nitsche & Augmented Lag & Mortar & Nodal \\
\hline \hline Max pressure relative error & $6.3 \cdot 10^{-3}$ & $9.6 \cdot 10^{-3}$ & $7.6 \cdot 10^{-3}$ & $7.4 \cdot 10^{-3}$ & $8.9 \cdot 10^{-2}$ \\
\hline Max gap $(\mathrm{mm})$ & $-2.6 \cdot 10^{-7}$ & $-2.9 \cdot 10^{-7}$ & $-3.9 \cdot 10^{-6}$ & $-3.9 \cdot 10^{-6}$ & $-7.6 \cdot 10^{-6}$ \\
\hline \hline
\end{tabular}

Table 2: Maximum of contact pressure relative error and gap for element-based integration. 
It is clear that segment-base integration is more precise and using element based integration technique generate a quadrature error. But this error remains still very small and can be considered acceptable in practice and it vanishes when refining the integration method (see Table 3). We observe from Table 2 that, despite the coarse mesh and the use of only 4 integration points per segment, the rate of quadrature error is low for Nitsche's method in its two versions. For both versions the accuracy is comparable even though the biased method seems to generate a lower error since the integration is performed only on one surface for this variant. Remark that changing the integration method does not affect the nodal method. The augmented lagrangian integral method and the mortar method have almost the same accuracy.

\begin{tabular}{cccccc}
\hline Approximation method & Biased Nitsche & Unbiased Nitsche & Augmented Lag & Mortar & Nodal \\
\hline \hline Max pressure relative error & $1.7 \cdot 10^{-3}$ & $2.2 \cdot 10^{-3}$ & $0.6 \cdot 10^{-3}$ & $0.6 \cdot 10^{-3}$ & $9.7 \cdot 10^{-2}$ \\
\hline Max gap $(\mathrm{mm})$ & $-0.7 \cdot 10^{-7}$ & $-1.5 \cdot 10^{-7}$ & $-1.6 \cdot 10^{-6}$ & $-1.6 \cdot 10^{-6}$ & $-7.5 \cdot 10^{-6}$ \\
\hline \hline
\end{tabular}

Table 3: Maximum of contact pressure relative error and gap for element-based integration and a finer inegration scheme(8 integration points).

In Table 3, we use the element based integration with 8 Gauss points per segment. The results show that for all integral formulations the integration error decreases significantly when refining numerical integration.

\subsection{Hertz contact}

We consider the case of a half-disc of radius $\mathrm{R}=10 \mathrm{~mm}$ pressed onto a plane elastic foundation. For the sake of simplicity, we consider a material law of Saint-Venant-Kirchhoff type. The elastic modulus is $E=10^{5}$ $\mathrm{MPa}$, and the Poisson ratio is set to $\nu=0.3$ for both bodies. The half-disc's top side is clamped and lowered vertically. For all the tests we compare the approximated solution to the Hertz one for a small rate of loading going from 0 to $0.5 \mathrm{~mm}$ in ten steps equally spaced. The tests were performed with both Lagrange linear and quadratic triangular finite elements and non maching interface meshes. The test is performed first with $\gamma_{0}=E$, $\theta=0$ and the unbiased variant.

The diagrams in Figure 4 correspond to the pressure profiles at the 10th load-step obtained with two and three quadrature points per element while Figure 5 corresponds to the penetration error measured on the contact surface (in $\mathrm{mm}$ ) when taking 3 integration points per element. Figure 5 allow to measure and locate the penetration on the contact surface with the proposed weak imposition of contact constraints. The vertical red arrows in Figure 4 correspond to values of the contact pressure field at quadrature points. The blue line represents the analytically calculated Hertz's pressure profile for the corresponding normal load obtained in the simulation. In both figures, diagrams in the left column correspond to a linear approximation of the displacement while the results in the right column refer to a quadratic one. 


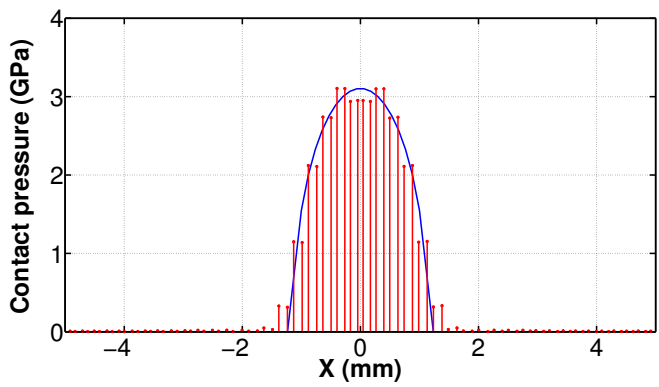

(a) Linear approximation (2 integration points)

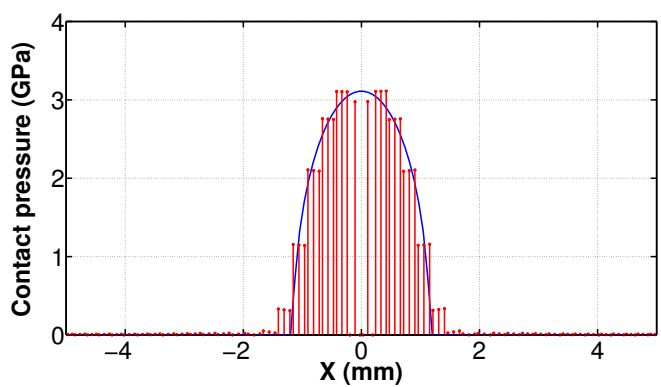

(c) Linear approximation (3 integration points)

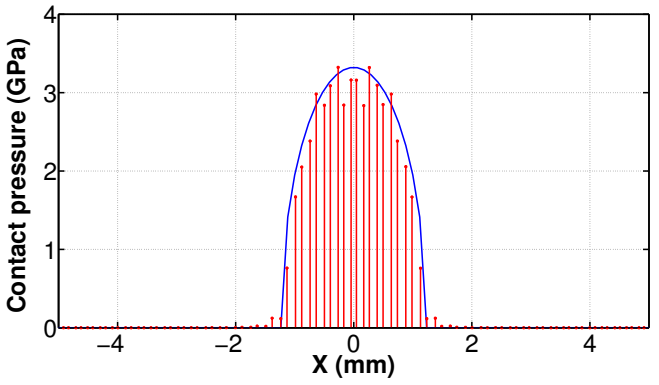

(b) Quadratic approximation (2 integration points)

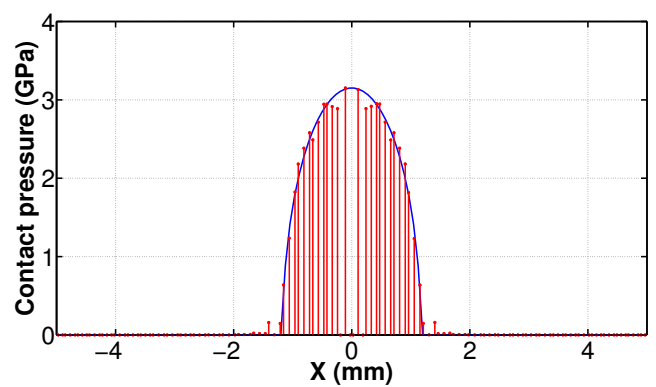

(d) Quadratic approximation (3 integration points)

Figure 4: Contact pressure for Hertz problem with unbiased Nitsche's method.

A first observation is that the method approximates well the Hertz solution: the exact pressure profile is reproduced and no problem is encountered with the release of the nodes for inactive contact elements. For linear approximation, two quadrature points per element edge appear to be sufficient for numerical integration whereas 3 points allow a better accuracy for the quadratic approximation. The linear approximation is accurate only for a sufficiently fine mesh because the pressure profile is not linear along the contact surface.

Figure 5 shows that the measured penetration is very low with linear and quadratic elements: less than $0.4 \%$ of the element size. To better see the convergence of this error when refining the mesh, we plot on Figure 6 the evolution of the measured penetration norm with the mesh size for Lagrange quadratic elements. This curve shows clearly that the gap error vanishes by refining the mesh. The order of convergence is approximately 2 in $L^{2}$-norm. Increasing the approximation order reduces as well the gap error.

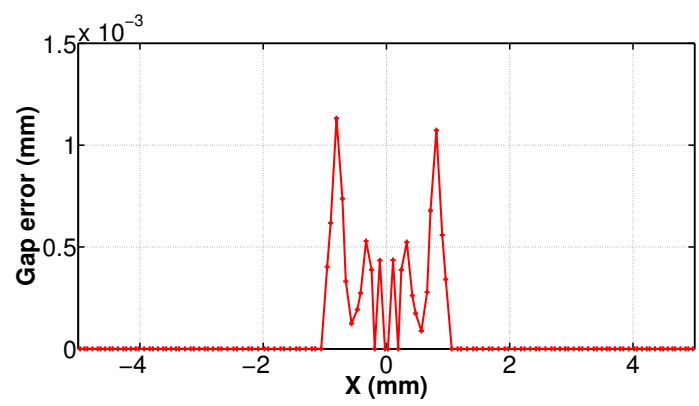

(a) Linear approximation

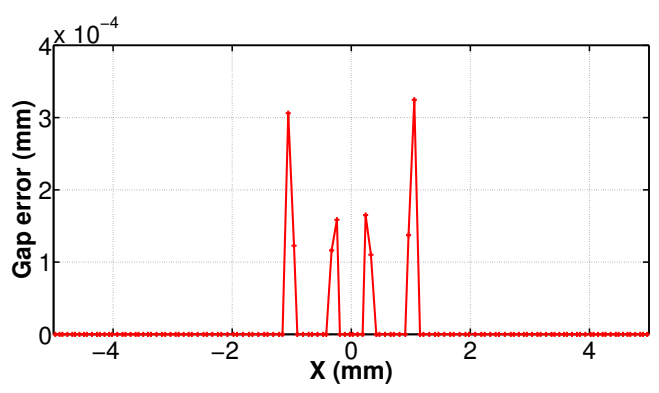

(b) Quadratic approximation

Figure 5: Gap error for Hertz problem with 3 integration points per element and unbiased Nitsche's method. 


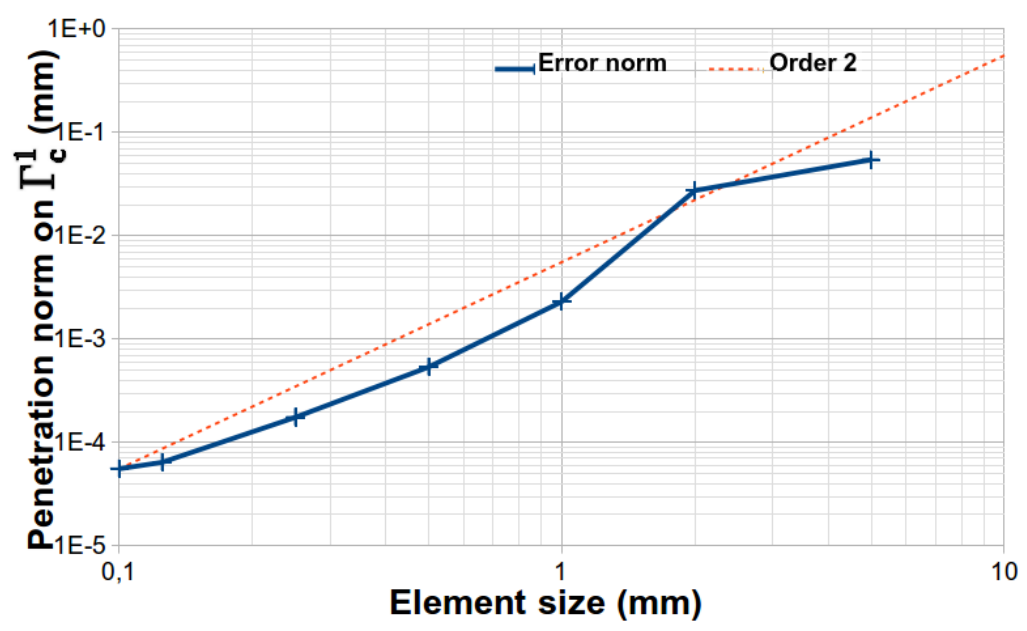

Figure 6: Evolution of gap error norm along $\Gamma_{c}^{1}$ with elements size.

The solution of this problem depends on Nitsche's parameter $\gamma$ in the sense that similarly to the small strain case studied in [9, 10] the convergence of the method is influenced by the parameter $\gamma_{0}$ and this dependency differs for different values of $\theta$. We provide in Table 4 the average (along the loading steps) of the pressure error and of the required Newton iterations yielding convergence with a maximum of 50 iterations for all steps, for different values of $\gamma_{0}$ and $\theta$. The test is performed for Lagrange quadratic triangular elements, with and without friction.

\begin{tabular}{|c|c|c|c|}
\hline \multicolumn{4}{|c|}{ Frictionless } \\
\hline 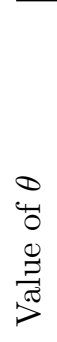 & 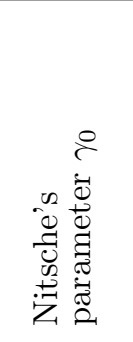 & 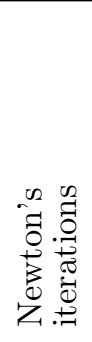 & 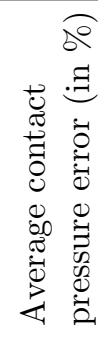 \\
\hline 0 & $\begin{array}{c}\frac{E}{100} \\
\mathrm{E} \\
100 \cdot E\end{array}$ & $\begin{array}{l}7.24 \\
3.5 \\
4.6\end{array}$ & $\begin{array}{l}17 \\
2.4 \\
2.7\end{array}$ \\
\hline-1 & $\begin{array}{c}\frac{E}{100} \\
\mathrm{E} \\
100 \cdot E\end{array}$ & $\begin{array}{l}4.8 \\
4.3 \\
4.6\end{array}$ & $\begin{array}{l}3.7 \\
3.0 \\
4.1\end{array}$ \\
\hline 1 & $\begin{array}{c}\frac{E}{100} \\
\mathrm{E} \\
100 \cdot E\end{array}$ & $\begin{array}{c}14.8 \\
26.8 \\
4\end{array}$ & $\begin{array}{c}54.8 \\
52.2 \\
4.2\end{array}$ \\
\hline
\end{tabular}

\begin{tabular}{|c|c|c|c|}
\hline \multicolumn{4}{|c|}{ Friction coefficient $\mathscr{F}=0.3$} \\
\hline 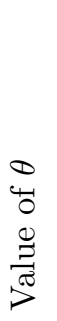 & 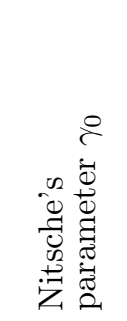 & 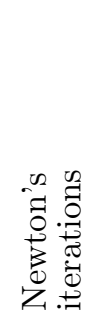 & 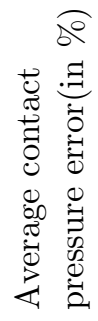 \\
\hline 0 & $\begin{array}{c}\frac{E}{100} \\
\mathrm{E} \\
100 \cdot E\end{array}$ & $\begin{array}{c}8.5 \\
4.6 \\
20.7\end{array}$ & $\begin{array}{c}14.4 \\
2.4 \\
6.8\end{array}$ \\
\hline-1 & $\begin{array}{c}\frac{E}{100} \\
\mathrm{E} \\
100 \cdot E\end{array}$ & $\begin{array}{l}5.3 \\
4.3 \\
9.2\end{array}$ & $\begin{array}{c}3.3 \\
3.1 \\
4\end{array}$ \\
\hline 1 & $\begin{array}{c}\frac{E}{100} \\
\mathrm{E} \\
100 \cdot E\end{array}$ & $\begin{array}{c}29.8 \\
30 \\
11.2\end{array}$ & $\begin{array}{l}59 \\
64 \\
4.1\end{array}$ \\
\hline
\end{tabular}

Table 4: Average of contact pressure error and number of Newton's iterations for Hertz contact.

As for small strain 9, 10 the influence of Nitsche's parameter $\gamma_{0}$ depends on $\theta$. It is remarkable that the skew-symmetric version $\theta=-1$ remains the most robust one and converges whatever is the value of $\gamma_{0}$. In this 
case the obtained pressure profile approximates well the theoretical one. This is also observed in 9 ] for small deformations and a mathematical proof is provided in that case. The symmetric version $\theta=1$ is the most sensitive one and it converges only when $\gamma_{0}$ is large enough. Comparing $\theta=0$ to $\theta=1$, we remark that the simple version $\theta=0$ is more robust regarding $\gamma_{0}$ since it converges for a wider range of values for $\gamma_{0}$. Nevertheless, convergence is lost when $\gamma_{0}$ becomes too small. The same behavior is observed in the frictional case. Additionally let us mention that when $\gamma_{0}$ is very large, convergence of the Newton algorithm is more difficult to achieve, especially for frictional contact. This is related to the fact that, when taking $\gamma_{0}$ too large, the problem becomes stiff and ill-conditioned (see, e.g., 32]).

Remark 3.1. Note that a recent attempt has been made to adapt a penalty-free Nitsche method originally analyzed in [5] to the Signorini problem (see [6]). However it does not correspond to the case $\gamma_{0}=0$ and there remains an additional numerical parameter, conversely to the case of Dirichlet boundary conditions which is parameter-free.

\subsection{Shallow ironing}

The third numerical example to be presented is the so-called shallow ironing test. An indenter with a circular arc shaped bottom edge is pressed against an elastic block and is forced to slide along the block length. This example can also be found for instance in [15, 29, 36. In this test we investigate the transmission of the force in vertical and horizontal directions when the contact surface evolves. We compare essentially the frictionless and the frictional case to test the accuracy of method when approximating a friction problem. Figure 7 shows the initial geometry with the different dimensions in mm. For the two contacting bodies a neo-Hookean material behavior is considered, with Young's moduli equal to $68.96 \cdot 10^{8} \mathrm{MPa}$ and $68.96 \cdot 10^{7} \mathrm{MPa}$ for the indenter and the block, respectively, and Poisson's ratio of 0.32 for both parts. The considered two-dimensional system is solved under the plane strain assumption. As in [29], we consider a quasi-static load. For $t \in[0,1]$, a vertical displacement of $1 \mathrm{~mm}$ is performed in 10 steps. Then, when $t \in[1,2]$, we perform an horizontal displacement along the block in 500 equal steps, each of $0.02 \mathrm{~mm}$. The three computed deformed configurations are presented in Figure 7 with a plot of the Von-Mises stress distribution, which demonstrate the finite deformations involved in the ironing process. This result and the curves of Figure 8 correspond to a friction coefficient $\mathscr{F}=0.3$ and quadratic rectangular finite elements. We use the simple version of the method $(\theta=0)$ and three quadrature points per segment for numerical integration. Since the material parameters of the two bodies are different, we consider, for the unbiased version, two different Nitsche's parameters $\gamma_{0}^{i}=E^{i}$. 

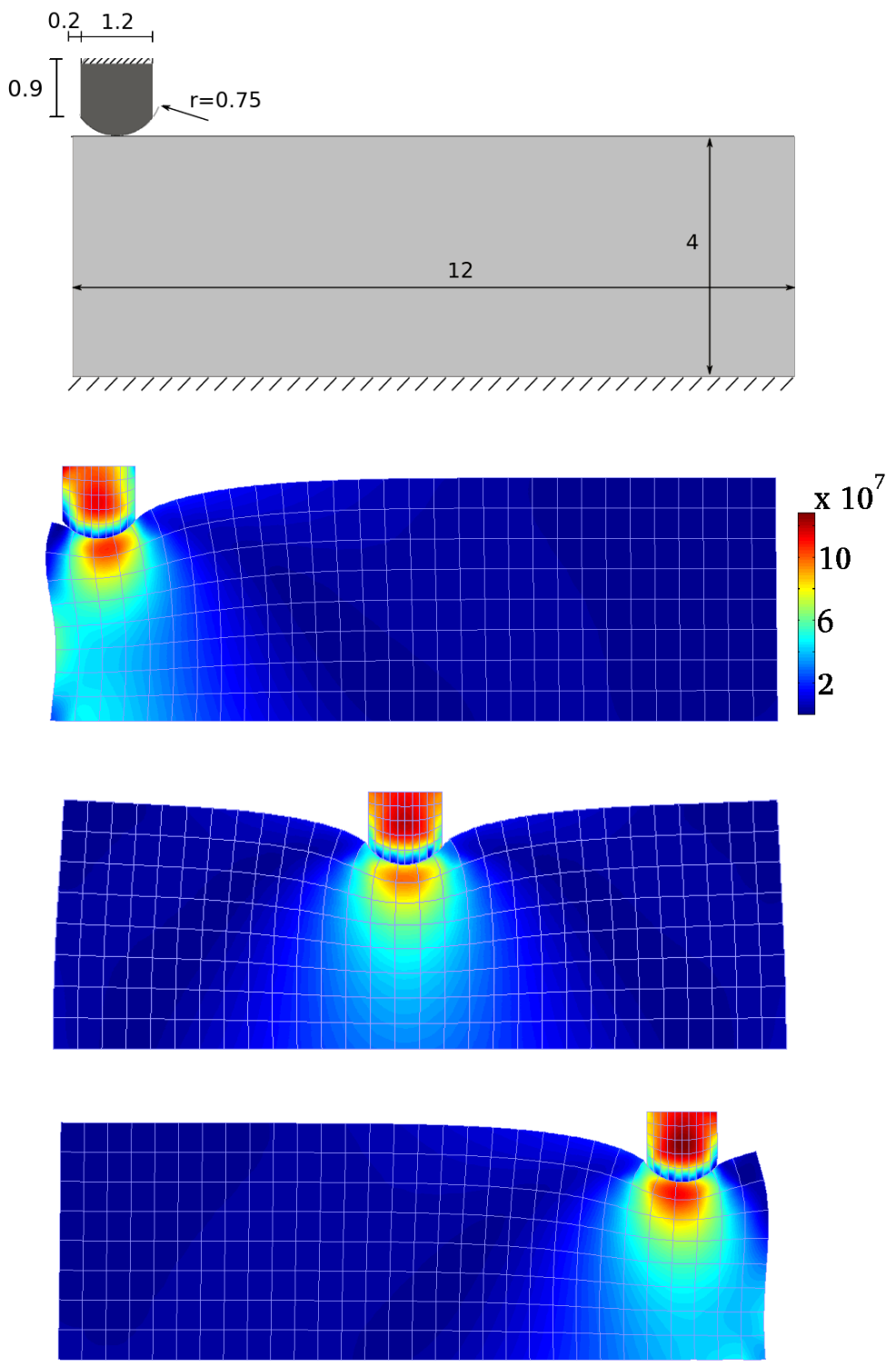

Figure 7: Initial geometry and deformed configuration of the shallow ironing example with contour plot of the Von-Mises stress in the frictionless case, at $t=1, t=1.5$ and $t=2$.

Figure 8 shows the evolution of the total horizontal and vertical force components between the contacting bodies during the whole simulation. During the first phase of pressing the indenter into the slab, the curves are smooth and the two bodies stick together. Starting the horizontal movement, the vertical as well as the horizontal reaction forces increase a bit until a limit is reached. At this stage the block starts sliding over the slab. An oscillation is observed for the vertical and horizontal reaction forces. This oscillation decreases when refining the mesh. This is due to the fact that the finite element mesh of the block has to slip around the right corner of the indenting body. Note however that these observed oscillations for vertical and horizontal force, even with only three quadrature points, remain still small, compared to similar results presented in [15. Comparing the results of the present study with those reported in [29, 15], one can note that the qualitative behavior is very well captured. The results are close to those obtained in [29] but there are important quantitative differences 


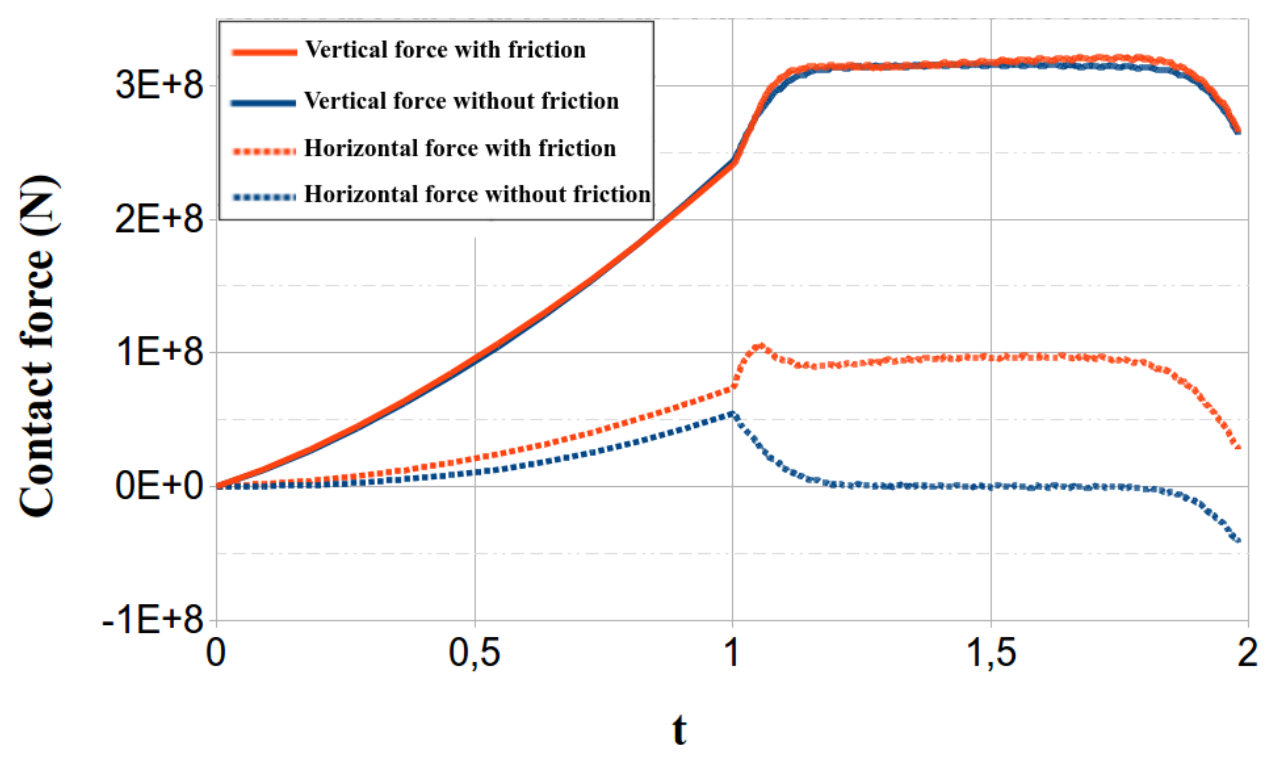

Figure 8: Evolution of the vertical and horizontal components of the contact forces for shallow ironing.

with [15. The vertical force in Figure 8 is slightly lower than in [15, while the reported horizontal force is significantly lower compared to the aforementioned reference. At time $1.5 \mathrm{~s}$ for example, the ratio between the horizontal and vertical force can be estimated to 0.53 , based on the results reported in [15] and 0.304 according to [29]. The obtained ratio with our Nitsche's unbiased method is 0.3014 , which is very close to the result of [29] and [36] where an integral augmented mixed method and a mrotar one are respectively used. The contact stress distribution is in agreement with common understanding of system mechanics and if we calculate the angle between the resulting stress vector along the contact surface at $t=1.5$ and the resulting surface normal, we get $15.36^{\circ}$. This appears to be very close to the friction angle of $16.7^{\circ}$ corresponding to the given coefficient of friction of 0.3 . For the frictionless case, a zero horizontal force is predicted correctly for the symmetric position at $t=1.5$, and it goes for negative values near the edge.

\subsection{Contact of an elastic half-ring}

In the fourth example, contact between an elastic ring undergoing large deformations and an elastic block is considered. As in reference [14, 36, both parts are assumed to exhibit neo-Hookean material behavior with Poisson's ratio equal to 0.3. As introduced in [36], in this test, the elastic half-ring is assembled from outer and inner rings with the same thickness of $5 \mathrm{~mm}$. The outer ring has a Young modulus of $10^{3} \mathrm{MPa}$ and the inner one is assumed to be 100 times stiffer. The Young modulus is of $300 \mathrm{MPa}$ for the block. The inner radius of the half-ring is equal to $90 \mathrm{~mm}$. The block is $260 \mathrm{~mm}$ long and $50 \mathrm{~mm}$ high. The rectangular block is fixed at its bottom edge, while the ends of the half-ring are horizontally fixed and vertically displaced by a total distance of $70 \mathrm{~mm}$ in 140 steps of size $0.5 \mathrm{~mm}$. Figure 9 shows the initial geometry and four deformed configurations at different time-steps. The deformations are obtained without and with friction coefficient $\mathscr{F}=0.5$. The coarsest mesh used in the calculations is made of 64 elements along the ring circumference and 1 element across each ring layer, while the block is discretized with 52 by 10 quadrilateral elements, in length and height directions respectively. On each body, Nitsche parameter will be equal to its Young modulus: $\gamma_{0_{R}}=E_{\text {Rext }}, \gamma_{0_{B}}=E_{\mathrm{B}}$. We consider the version $\theta=0$. 

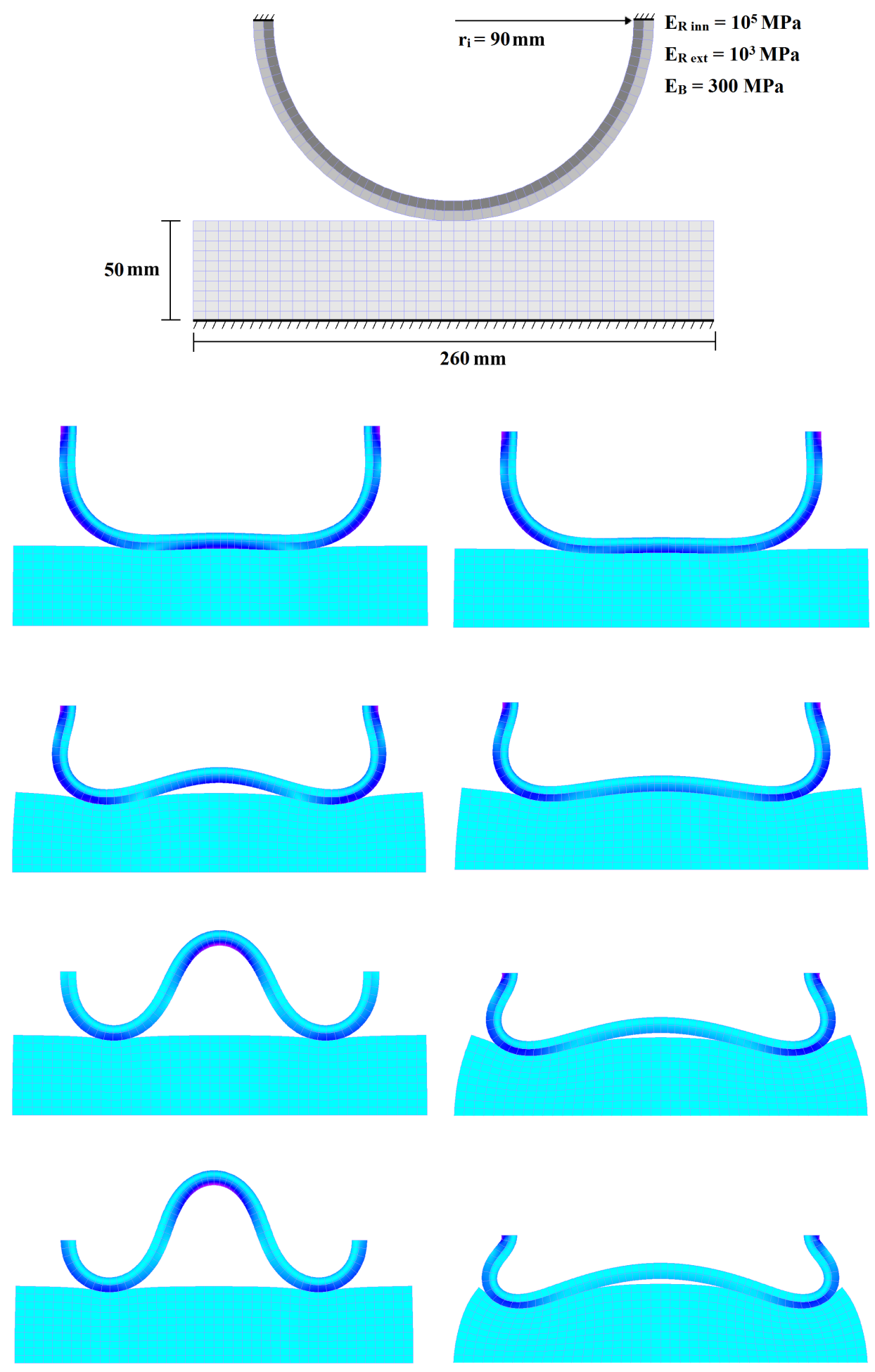

Figure 9: Deformation of the elastic half ring without friction (left) and with $\mathscr{F}=0.5$ (right) for $\mathbb{Q}_{2}$ elements, after a loading of $25,45,60$ and $70 \mathrm{~mm}$. 
This example allows us to test the accuracy of the Nitsche method in the case of heterogeneous materials and high friction forces. To compare the computed deformation with previous results from other methods, we measure the vertical displacement of the ring's mid-point. This displacement along the load steps is plotted in Figure 10, both for frictionless and frictional contact.

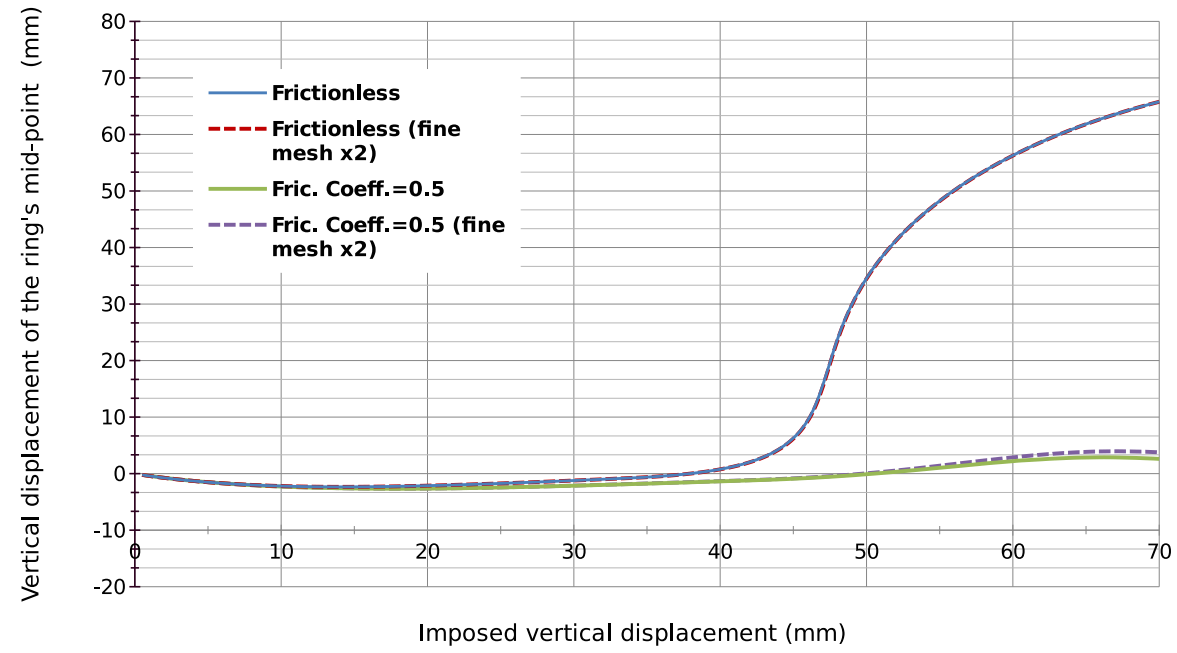

Figure 10: Vertical displacement of the half-ring middle point for different mesh sizes.

Figure 9 shows that the loaded half-ring compresses initially the elastic block on its central contact surface, as expected. At this stage the frictional and frictionless cases are quite similar and the central mid-point of the half-ring moves downwards. This is observable on Figure 10 until an amount of imposed displacement of 20 to $25 \mathrm{~mm}$ is reached. This corresponds to the first deformed configuration in Figure 9 . Subsequently, the tracked point is lifted progressively until $45 \mathrm{~mm}$ of displacement. Then, in the interval between $45 \mathrm{~mm}$ and $60 \mathrm{~mm}$, the lifting speed of the half-ring middle point peaks in absence of friction when it remains low in the frictional case because of extensive sliding between the ring and the block. In the remaining part of the simulation the tracked point keep on moving up, but with a lower speed in both cases. The results with coarse and refined meshes are very similar for the frictionless case, and also for the frictional one but only until $50 \mathrm{~mm}$ of displacement. In the last $20 \mathrm{mms}$ of the simulation, a remarkable difference between the two approximations is observed, when considering friction. This could be due to the important sliding forces since we do not get that error in the frictionless case. As for Hertzian contact, Nitsche's parameter $\gamma_{0}$ needs to be large enough for stability and convergence, but when it is too large the problem stiffens: some elements are inverted and convergence is difficult to achieve. The optimal values of $\gamma_{0}$ on each material are those near its Young modulus $E$. For $\gamma_{0}=E$ we obtain an average of Newton's iterations of 4.45 for frictionless contact and 4.44 for frictional contact. With a fine mesh the convergence speed is similar with a slight difference for the frictional case since the average in that case is 5.05.

To test the method with large contrast of stiffness between the contacting bodies, we measure the average of Newton's iterations with different values of the ratio $E_{\mathrm{Rext}} / E_{\mathrm{B}}$ in the frictional case. The Young modulus of the inner ring is constant $\left(=10^{5} \mathrm{MPa}\right)$ and as for previous tests, Nitsche's parameter is equal to the Young modulus on each body. 


\begin{tabular}{ccccccccc}
\hline$E_{\text {Rext }} / E_{\mathrm{B}}$ & $10^{-5}$ & $10^{-3}$ & $10^{-2}$ & $10^{-1}$ & 1 & 10 & $10^{2}$ & $10^{3}$ \\
\hline Average of Newton's iterations & No convergence & 6,43 & 6,07 & 6,35 & 7,66 & 6,36 & 5,73 & No convergence \\
\hline
\end{tabular}

Table 5: Average of Newton's iterations with different values of $E_{\text {Rext }} / E_{\mathrm{B}}$.

We observe that a large difference of stiffness does not influence the accuracy of the unbiased method. The convergence is obtained with a close number of iterations for different values of the stiffness ratio. We loose convergence only when going to extreme values because, when the block is too much flabby, some elements are inverted; and when it is too rigid the problem becomes stiff and ill-conditioned.

\subsection{Crossed Tubes with self-contact}

The last numerical example is the crossed tubes test. In this example we simulate contact between two crossed hollow elastic cylinders. Each of the tubes has an outer diameter of $24 \mathrm{~mm}$, a wall thickness equal to 0.8 $\mathrm{mm}$ and a length equal to $100 \mathrm{~mm}$. Neo-Hookean material behavior is considered for both tubes, with material parameters corresponding to Poisson's ratio equal to 0.3 and Young moduli of $E_{1}=10^{5} \mathrm{MPa}$ for the lower tube and $E_{2}=10^{4} \mathrm{MPa}$ for the upper one. The tubes are forced into contact through Dirichlet conditions applied at their ends. The upper tube is displaced vertically for a total distance of $40 \mathrm{~mm}$ divided into 80 equal load steps. Since the enforced displacement is large, the deformations of the tubes are large and we observe a self-contact configuration on the less rigid tube. So this test allows us to validate our method in case of self-contact. Since the geometry as well as the boundary conditions are symmetric, it is sufficient to model only one quarter of the considered structure. The actually modeled portion of each tube is colored in Figure 11 and it is discretized using 16 by 24 by 2 three-dimensional elements in the length, circumferential and radial directions respectively.

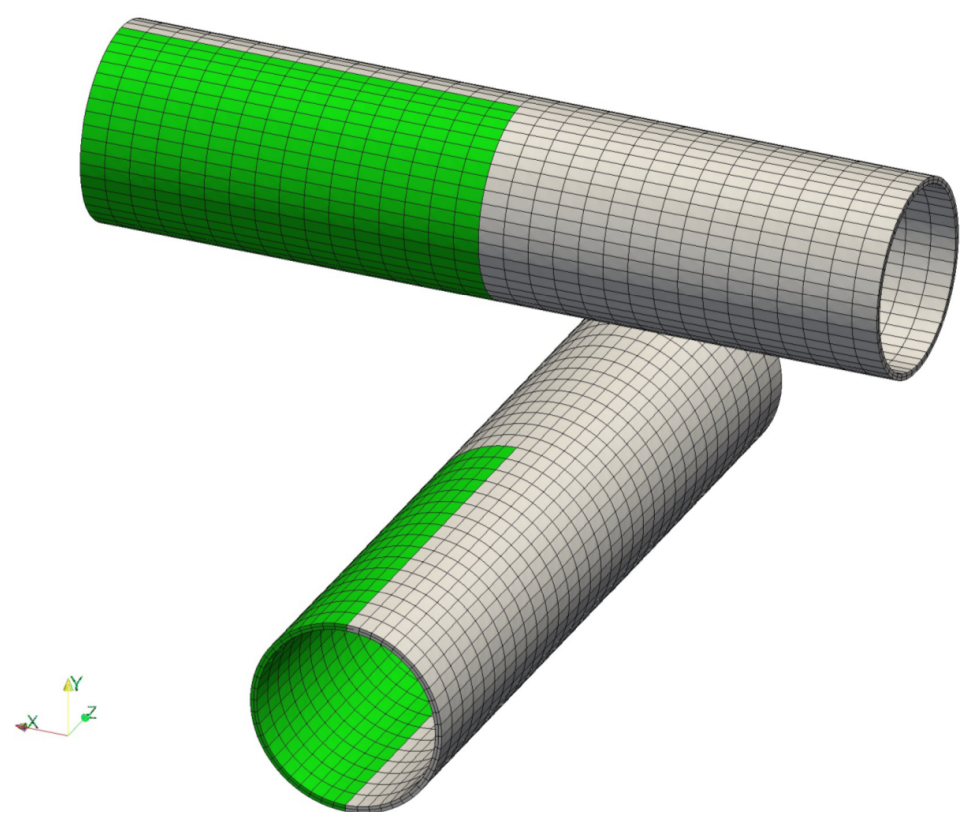

Figure 11: Geometry and mesh of the crossed tubes in their initial configuration.

The presented solution is based on an approximation of the geometry and of the displacement with quadratic hexahedral elements. The unbiased Nitsche method with $\theta=0$ is considered to deal with self-contact. The results of Figure 12 correspond to the frictionless case with a Nitsche's parameter $\gamma_{0}=E_{1}$ for the lower tube and $\gamma_{0}=E_{2}$ for the upper one. Figure 12 depicts the calculated deformed configurations for the 30th, 60th 
and 80th load steps. Figure 13 shows the evolution along the loading steps of required Newton's iterations for the frictionless case and for a friction coefficient $\mathscr{F}=0.3$. Despite the increase of the required iterations when self-contact occurs, Newton's algorithm converges in general within a few iterations. The required iterations number for convergence increases from the 45th load step. This is due to the onset of self-contact.
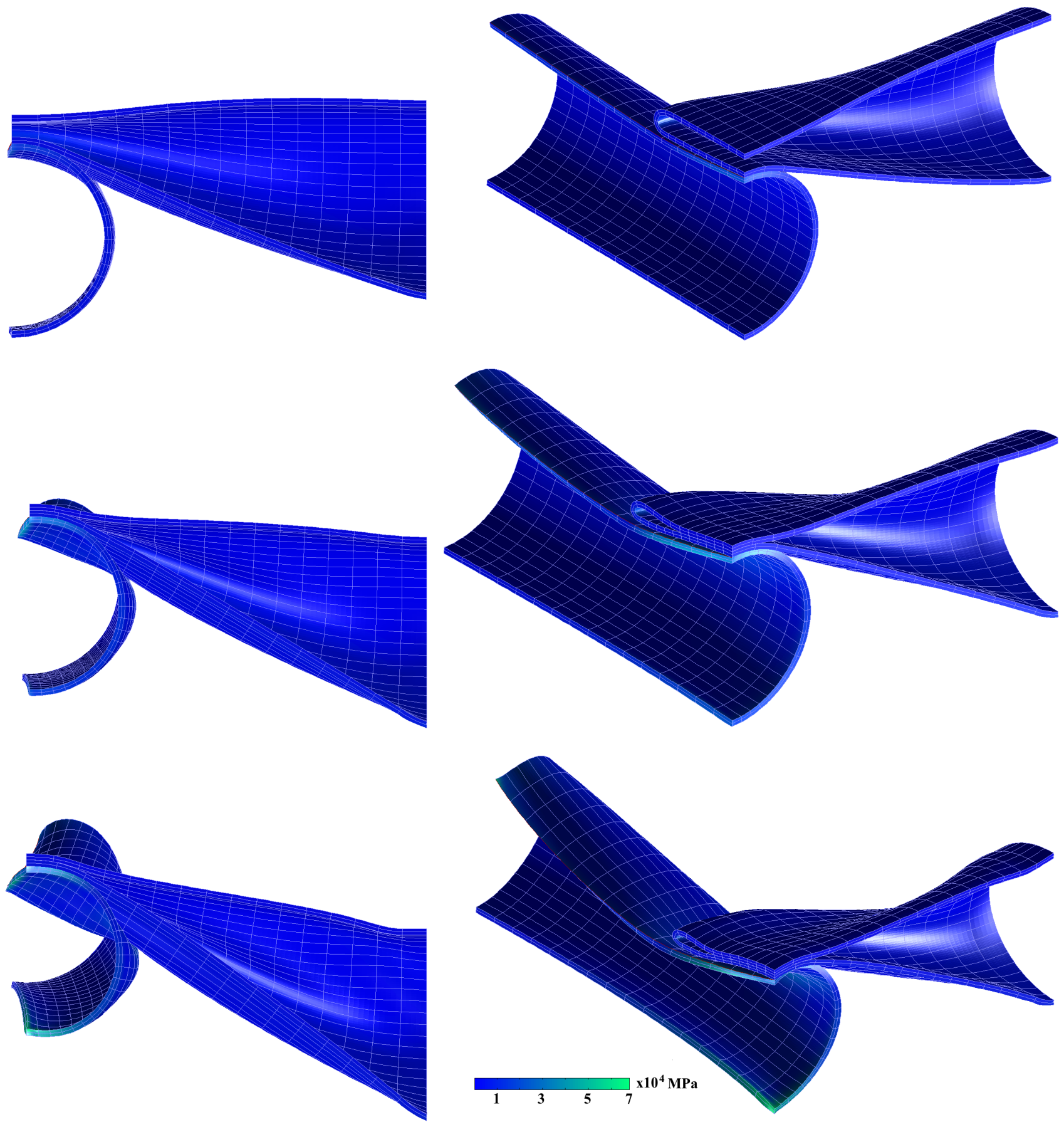

Figure 12: Deformation and Von-Mises contour plot of the two crossed tubes test without friction for a loading of 20, 30 and $40 \mathrm{~mm}$. 


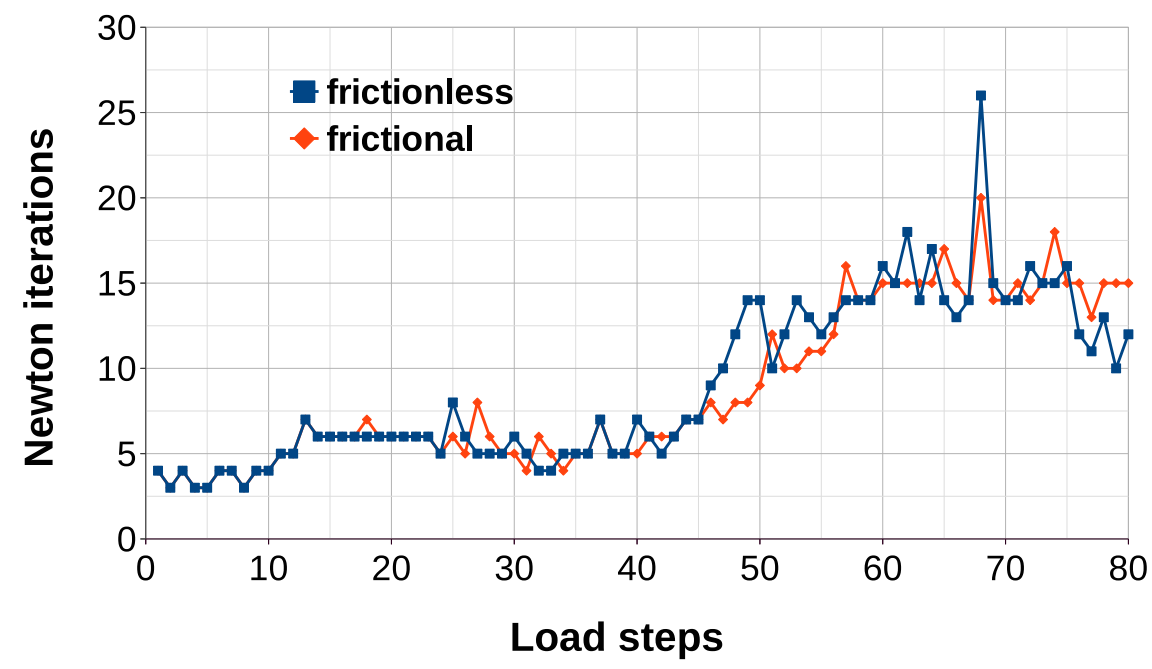

Figure 13: Crossed tubes test: required Newton's iterations per load step with $(\mathscr{F}=0.3)$ and without friction.

\subsection{Projection and ray-tracing}

For contact problems, the choice of mapping strategy influences directly the performance and the robustness of the method. In this last part we compare the two presented mappings from a numerical viewpoint. The comparison is made through two tests: two-dimensional Hertz's contact and three-dimensional crossed tubes.

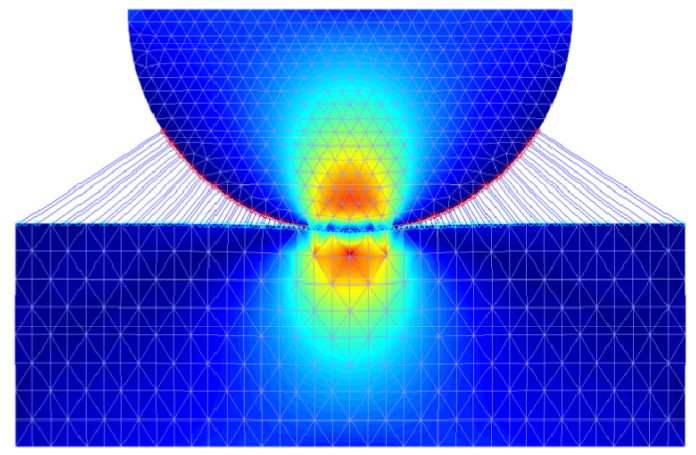

(a) Ray-tracing

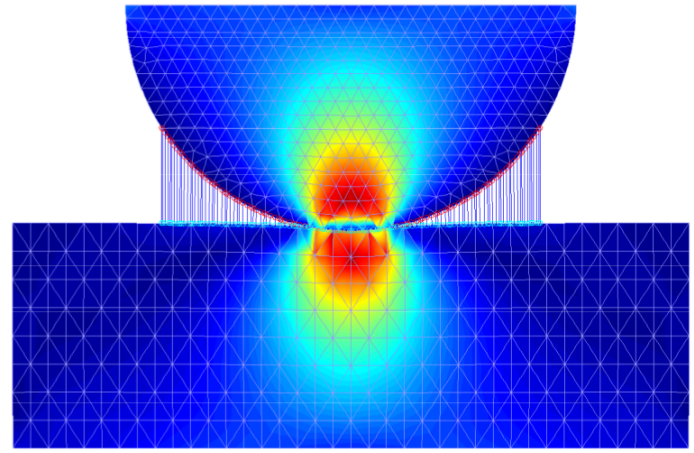

(b) Projection

Figure 14: Illustration of ray-tracing and projection from the upper interface to the lower one and Von mises pressure profiles for Hertz contact.

At first, we illustrate in Figure 14 the difference between ray-tracing and projection for Hertz problem and we present the deformation and the effort distribution obtained by using the two mappings. The two strategies solve well Hertz contact problem, but to compare them, we provide for each one the average number of Newton iterations yielding convergence as well as the mean pressure error along the ten steps of loading. The test is performed with different values of $\theta$, with and without friction. For sake of briefness, we choose only the value of $\gamma_{0}$ equal to $E$ and the unbiased version of the method. 


\begin{tabular}{|c|c|c|c|}
\hline \multicolumn{4}{|c|}{ Ray-tracing } \\
\hline $\begin{array}{l}0 \\
4 \\
0 \\
0 \\
\frac{1}{7} \\
\frac{\pi}{7}\end{array}$ & 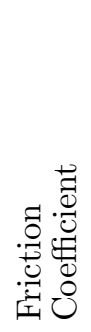 & 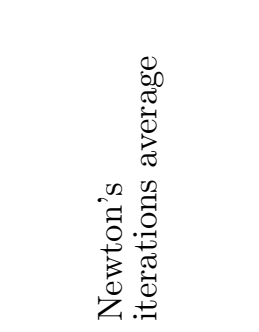 & 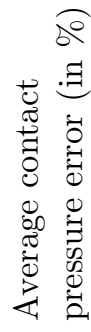 \\
\hline \multirow[t]{2}{*}{0} & 0 & 2.7 & 3.34 \\
\hline & 0.3 & 2.8 & 3.4 \\
\hline \multirow[t]{2}{*}{-1} & 0 & 3.4 & 3.36 \\
\hline & 0.3 & 3.3 & 3.46 \\
\hline \multirow[t]{2}{*}{1} & 0 & No convergence & - \\
\hline & 0.3 & No convergence & - \\
\hline
\end{tabular}

\begin{tabular}{|c|c|c|c|}
\hline \multicolumn{4}{|c|}{ Projection } \\
\hline 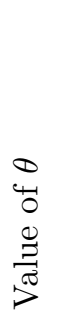 & 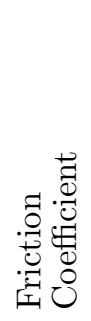 & 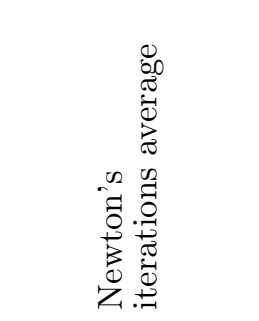 & 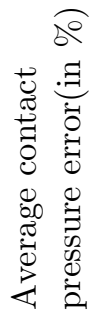 \\
\hline \multirow[t]{2}{*}{0} & 0 & 5.9 & 3.39 \\
\hline & 0.3 & 6.5 & 3.35 \\
\hline \multirow[t]{2}{*}{-1} & 0 & 6.3 & 3.36 \\
\hline & 0.3 & 6.5 & 3.45 \\
\hline \multirow[t]{2}{*}{1} & 0 & No convergence & - \\
\hline & 0.3 & No convergence & - \\
\hline
\end{tabular}

Table 6: Mean contact pressure and number of Newton's iterations for projection and ray-tracing strategy, for the Hertz test with $\gamma_{0}=E$ and the unbiased Nitsche's method.

A first observation is that the accuracy of the approximation is the same for the two mappings, meaning that the choice of the mapping does not influence strongly the quality of the solution. When $\theta=1$ we do not reach convergence since $\gamma_{0}$ is not large enough. So the two strategies seem to have the same response regarding Nitsche's parameter. In addition, the difference in terms of Newton's iterations is clearly observable for different values of $\theta$. Ray-tracing allows a convergence twice faster than projection. This may be due to the non-exactitude of the tangent problem for projection in which we neglected the directional derivative of $n_{y}$ term. A similar difference of convergence speed is observed for the crossed tubes test in Figure 15. If we apart the influence of self-contact, the number of iterations is almost two times higher for projection. The smoothness of the contact surfaces in both tests does not allow to study robustness regarding some special cases detailed in 29].

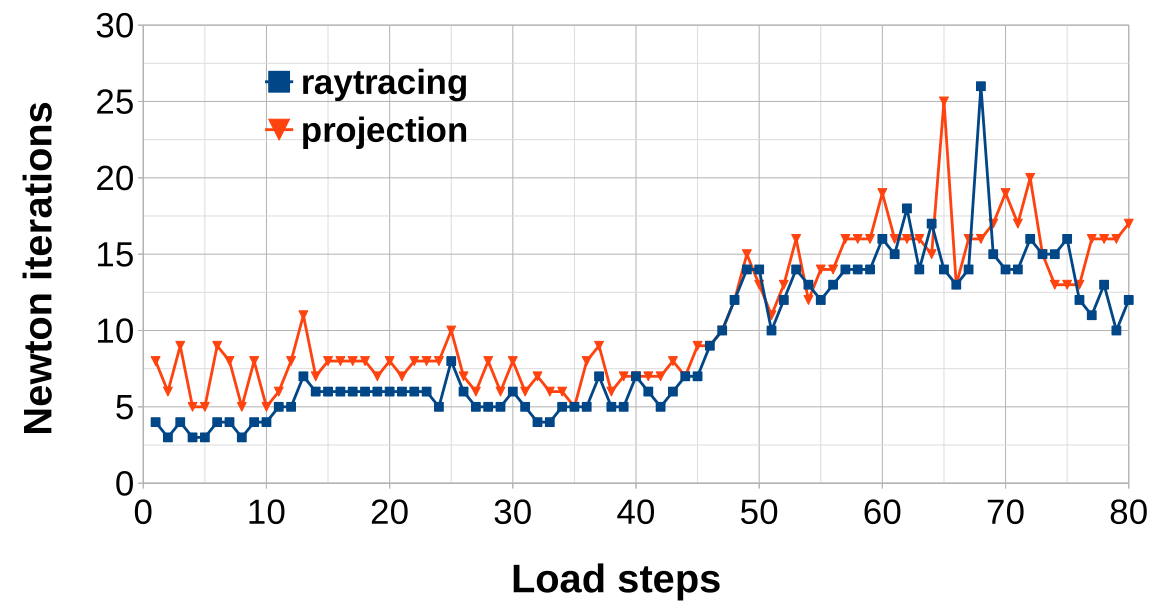

Figure 15: Required Newton's iterations per load step for the crossed tubes test without friction, for the ray-tracing and projection mapping strategies. 


\section{Conclusion}

Nitsche's method is a promising formulation to treat interface conditions in a consistent weak sens. Adapted to the contact problem the method offers several theoretical and numerical advantages in the small strain framework. The aim of this work is to extend the method to the large strain case. We proposed an extension of Nitsche's method to contact and friction in the large strain framework. We derived an unbiased formulation that can be more amenable to self-contact and multi-body contact. A generalization parameter $\theta$ is considered to cover an entire set of methods with different numerical properties. The method is constructed independently of the mapping function, but its performance depends on the used mapping. Therefore, we gave a brief comparison of two mapping strategies: projection and ray-tracing. Being an integral formulation, the performance of Nitsche's method depends on the used quadrature scheme. We made the choice of using the classic Gauss schemes over the original elements without cutting. This choice was briefly argued, but, still, the effect of numerical integration for Nitsche's method is an interesting perspective of study. In this work, we used an hyper-elastic material, but, since the behavior law is used, extending the method to inelastic behavior may need some adaptations since state variables are typically calculated and stored at quadrature points on the interior of the elements. In a Nitsche approach, such fields will also need to be computed and stored on the surface. Therefore an extension to inelastic materials could be considered in forthcoming studies.

\section{Acknowledgements}

We thank the two anonymous referees for their helpful comments that allowed to improve the paper. We would like also to sincerely thank the company "Manufacture Française des Pneumatiques Michelin" for the financial and technical support. We thank, as well, Région Bourgogne Franche-Comté for partial funding ("Convention Région 2015C-4991. Modèles mathématiques et méthodes numériques pour l'élasticité non-linéaire").

\section{AppendixA. Directional derivative of the stress tensor}

To make explicit each term in formulation (15), we need first the following results on the directional derivatives of deformation tensors, that are obtained after simple computations (see, e.g. [16, Chapter 14]):

Proposition AppendixA.1. The directional derivatives of $\mathbf{F}$ and $\mathbf{E}$ are:

$$
\begin{aligned}
& \mathcal{D} \mathbf{F}(u)[\delta u]=\nabla_{X}(\delta u), \\
& \mathcal{D} \mathbf{E}(u)[\delta u]=\operatorname{sym}\left(\mathbf{F}^{T}(u) \nabla_{X}(\delta u)\right)=\mathbf{F}^{T}(u) \boldsymbol{\epsilon}(\delta u) \mathbf{F}(u),
\end{aligned}
$$

where sym $(\cdot)$ denotes the symmetric part of a second order tensor.

The computation of the directional derivatives of stress tensors $\hat{\boldsymbol{\sigma}}$ and $\mathbf{S}$ is more involved and we recall their expression below (see as well [16, Chapter 14]):

Proposition AppendixA.2. The directional derivatives of $\mathbf{S}$, resp. $\hat{\boldsymbol{\sigma}}$, are:

$$
\begin{aligned}
\mathcal{D} \mathbf{S}(u)[\delta u] & =\mathcal{C}(u): \mathbf{F}^{T}(u) \nabla_{X}(\delta u)=\mathcal{C}(u): \mathbf{F}^{T}(u) \boldsymbol{\epsilon}(\delta u) \mathbf{F}(u) \\
\mathcal{D} \hat{\boldsymbol{\sigma}}(u)[\delta u] & =\nabla_{X}(\delta u) \mathbf{S}(u)+\mathbf{F}(u)\left(\mathcal{C}(u): \mathbf{F}^{T}(u) \nabla_{X}(\delta u)\right) .
\end{aligned}
$$

Proof: First, for an hyperelastic law, there holds in fact $\mathbf{S}(u)=\mathbf{S}(\mathbf{E}(u))$ and we apply the chain rule:

$$
\mathcal{D} \mathbf{S}(u)[\delta u]=\mathcal{D} \mathbf{S}(\mathbf{E}(u))[\mathcal{D} \mathbf{E}(u)[\delta u]]=\frac{\partial \mathbf{S}}{\partial \mathbf{E}}(u): \mathcal{D} \mathbf{E}(u)[\delta u] .
$$

Since $\mathcal{C}=\frac{\partial \mathbf{S}}{\partial \mathbf{E}}$ and using Proposition AppendixA.1 we get:

$$
\mathcal{D} \mathbf{S}(u)[\delta u]=\mathcal{C}(u): \operatorname{sym}\left(\mathbf{F}^{T}(u) \nabla_{X}(\delta u)\right) .
$$

Then A.1 is obtained with the above formula and the symmetry properties of $\mathcal{C}$. 
Using the relationship $\hat{\boldsymbol{\sigma}}=\mathbf{F S}$ and applying the product rule yield, for $\mathcal{D} \hat{\boldsymbol{\sigma}}(u)[\delta u]$ :

$$
\mathcal{D} \hat{\boldsymbol{\sigma}}(u)[\delta u]=\mathcal{D} \mathbf{F}(u)[\delta u] \mathbf{S}(u)+\mathbf{F}(u) \mathcal{D} \mathbf{S}(u)[\delta u] .
$$

We use A.1 and once again Proposition AppendixA.1 to obtain A.2.

As an example, suppose that the constitutive law is those of a Saint-Venant-Kirchhoff material, i.e., that:

$$
W(\mathbf{E})=\frac{\lambda}{2}(\operatorname{tr}(\mathbf{E}))^{2}+\mu \operatorname{tr}\left(\mathbf{E}^{2}\right),
$$

where $\lambda$ and $\mu$ are material parameters, see, e.g., 4, Chapter 5]. The associated second Piola-Kirchhoff stress tensor and elasticity tensor are:

$$
\mathbf{S}=\lambda \operatorname{tr}(\mathbf{E}) \mathbf{I}+2 \mu \mathbf{E}, \quad \mathcal{C}=\lambda \mathbf{I} \otimes \mathbf{I}+2 \mu \mathcal{I} .
$$

Let us detail the expression

$$
\begin{aligned}
\mathcal{D} \hat{\boldsymbol{\sigma}}(u)[\delta u] & =\nabla_{X}(\delta u) \mathbf{S}(u)+\mathbf{F}(u)\left(\mathcal{C}(u): \mathbf{F}^{T}(u) \nabla_{X}(\delta u)\right) \\
& =\nabla_{X}(\delta u)(\lambda \operatorname{tr}(\mathbf{E}(u)) \mathbf{I}+2 \mu \mathbf{E}(u))+\mathbf{F}(u)\left((\lambda \mathbf{I} \otimes \mathbf{I}+2 \mu \mathcal{I}): \mathbf{F}^{T}(u) \nabla_{X}(\delta u)\right) .
\end{aligned}
$$

We compute separately

$$
\mathbf{I} \otimes \mathbf{I}: \mathbf{F}^{T}(u) \nabla_{X}(\delta u)=\left(\mathbf{I}: \mathbf{F}^{T}(u) \nabla_{X}(\delta u)\right) \mathbf{I}=\operatorname{tr}\left(\mathbf{F}^{T}(u) \nabla_{X}(\delta u)\right) \mathbf{I},
$$

and

$$
\mathcal{I}: \mathbf{F}^{T}(u) \nabla_{X}(\delta u)=\operatorname{sym}\left(\mathbf{F}^{T}(u) \nabla_{X}(\delta u)\right)
$$

This yields

$$
\begin{aligned}
\mathcal{D} \hat{\boldsymbol{\sigma}}(u)[\delta u]= & \nabla_{X}(\delta u)(\lambda \operatorname{tr}(\mathbf{E}(u)) \mathbf{I}+2 \mu \mathbf{E}(u)) \\
& +\mathbf{F}(u)\left(\lambda \operatorname{tr}\left(\mathbf{F}^{T}(u) \nabla_{X}(\delta u)\right) \mathbf{I}+2 \mu \operatorname{sym}\left(\mathbf{F}^{T}(u) \nabla_{X}(\delta u)\right)\right) \\
= & \lambda\left(\operatorname{tr}(\mathbf{E}(u)) \nabla_{X}(\delta u)+\operatorname{tr}\left(\mathbf{F}^{T}(u) \nabla_{X}(\delta u)\right) \mathbf{F}(u)\right) \\
& +2 \mu\left(\nabla_{X}(\delta u) \mathbf{E}(u)+\mathbf{F}(u) \operatorname{sym}\left(\mathbf{F}^{T}(u) \nabla_{X}(\delta u)\right)\right) .
\end{aligned}
$$

We finally obtain $\mathcal{D} \hat{\sigma}_{N}(u)[\delta u]$ using relationship:

$$
\mathcal{D} \hat{\sigma}_{N}(u)[\delta u]=\mathcal{D} \hat{\boldsymbol{\sigma}}(u)[\delta u] N,
$$

since $\hat{\sigma}_{N}=\hat{\boldsymbol{\sigma}} N$. The same process can be applied for various constitutive laws.

\section{References}

[1] Alart, P., Curnier, A., 1988. A generalized Newton method for contact problems with friction. J. Mec. Theor. Appl. 7 (1), 67-82.

[2] Annavarapu, C., Hautefeuille, M., Dolbow, J. E., 2014. A Nitsche stabilized finite element method for frictional sliding on embedded interfaces. Part I: Single interface. Comp. Meth. Appl. Mech. Eng. 268, $417-436$.

[3] Baillet, L., Sassi, T., 2005. Mixed finite element formulation in large deformation frictional contact problem. Rev. EuropÃ@enne Élém. Finis 14 (2-3), 287-304.

[4] Bonet, J., Wood, R. D., 2008. Nonlinear continuum mechanics for finite element analysis. Cambridge University Press, Cambridge (UK), New York. 
[5] Burman, E., 2012. A penalty-free nonsymmetric Nitsche-type method for the weak imposition of boundary conditions. SIAM J. Numer. Anal. 50 (4), 1959-1981.

[6] Burman, E., Hansbo, P., Larson, M. G., 2016. The Penalty Free Nitsche Method and Nonconforming Finite Elements for the Signorini Problem. arXiv preprint arXiv:1609.03745.

[7] Chouly, F., 2014. An adaptation of Nitsche's method to the tresca friction problem. J. Math. Anal. App. 411, 329-339.

[8] Chouly, F., Hild, P., 2013. Nitsche-based method for unilateral contact problems: numerical analysis. SIAM J. Numer. Anal. 51, 1295-1307.

[9] Chouly, F., Hild, P., Renard, Y., 2015. Symmetric and non-symmetric variants of Nitsche's method for contact problems in elasticity: theory and numerical experiments. Math. Comp. 84, 1089-1112.

[10] Chouly, F., Mlika, R., Renard, Y., 2016. An unbiased Nitsche's approximation of the frictional contact between two elastic structures. Submitted, Available on HAL as hal-01240068.

[11] Crisfield, M. A., 2000. Re-visiting the contact patch test. Internat. J. Numer. Methods Eng. 48, 435-449.

[12] Curnier, A., He, Q., Klarbring, A., 1995. Continuum mechanics modelling of large deformation contact with friction. Contact Mech., 145-158.

[13] Farah, P., Popp, A., Wall, W. A., 2015. Segment-based vs. element-based integration for mortar methods in computational contact mechanics. Comp. Mech. 55 (1), 209-228.

[14] Fischer, K. A., Wriggers, P., 2005. Frictionless 2D contact formulations for finite deformations based on the mortar method. Comp. Mech. 36 (3), 226-244.

[15] Fischer, K. A., Wriggers, P., 2006. Mortar based frictional contact formulation for higher order interpolations using the moving friction cone. Comp. Meth. Appl. Mech. Eng. 195, 5020-5036.

[16] Fortin, A., Garon, A., 2011. Les éléments finis: de la théorie à la pratique. Université Laval.

[17] Haikal, G., Hjelmstad, K., 2007. A finite element formulation of non-smooth contact based on oriented volumes for quadrilateral and hexahedral elements. Comp. Meth. Appl. Mech. Eng. 196, 4690 - 4711.

[18] Hansbo, A., Hansbo, P., 2004. A finite element method for the simulation of strong and weak discontinuities in solid mechanics. Comp. Meth. Appl. Mech. Eng. 193, 3523-3540.

[19] Hild, P., 2000. Numerical implementation of two nonconforming finite element methods for unilateral contact. Comp. Meth. Appl. Mech. Eng. 184, 99-123.

[20] Hild, P., Renard, Y., 2010. A stabilized lagrange multiplier method for the finite element approximation of contact problems in elastostatics. Numer. Math. 115, 101-129.

[21] Kikuchi, N., Oden, J. T., 1988. Contact problems in elasticity: a study of variational inequalities and finite element methods. Society for Industrial and Applied Mathematics (SIAM).

[22] Konyukhov, A., Schweizerhof, K., 2013. Computational Contact Mechanics. Lecture Notes in Applied and Computational Mechanics. Springer, Berlin.

[23] Laursen, T. A., 2002. Computational contact and impact mechanics. Springer, Berlin.

[24] Laursen, T. A., Simo, J., 1993. A continuum-based finite element formulation for the implicit solution of multibody, large deformation frictional contact problems. Internat. J. Numer. Meth. Eng. 36, 451-3485.

[25] McDevitt, T. W., Laursen, T. A., 2000. A mortar-finite element formulation for frictional contact problems. Internat. J. Numer. Meth. Eng. 48, 1525-1547. 
[26] Nitsche, J., 1971. Über ein Variationsprinzip zur Lösung von Dirichlet-Problemen bei Verwendung von Teilräumen, die keinen Randbedingungen unterworfen sind. Abh. Math. Semin. Univ. Hamb. 36, 9-15.

[27] Oliver, J., Hartmann, S., Cante, J., Weyler, R., Hernández, J. A., 2009. A contact domain method for large deformation frictional contact problems. Part 1: Theoretical basis. Comp. Meth. Appl. Mech. Eng. 198, $2591-2606$.

[28] Popp, A., Wohlmuth, B., Gee, M., Wall, W., 2012. Dual quadratic mortar finite element methods for 3D finite deformation contact. SIAM J. Sci. Comp. 34 (4), B421-B446.

[29] Poulios, K., Renard, Y., 2015. An unconstrained integral approximation of large sliding frictional contact between deformable solids. Comp. Struct. 153, 75-90.

[30] Puso, M. A., Laursen, T. A., 2002. A 3D contact smoothing method using Gregory patches. Internat. J. Numer. Meth. Eng. 54, 1161-1194.

[31] Puso, M. A., Laursen, T. A., 2004. A mortar segment-to-segment contact method for large deformation solid mechanics. Comp. Meth. Appl. Mech. Eng. 193, 601-629.

[32] Renard, Y., 2013. Generalized Newton's methods for the approximation and resolution of frictional contact problems in elasticity. Comp. Meth. Appl. Mech. Eng. 256, 38-55.

[33] Sauer, R. A., DeLorenzis, L., 2015. An unbiased computational contact formulation for 3D friction. Internat. J. Numer. Meth. Eng. 101 (4), 251-280.

[34] Simo, J., Laursen, T. A., 1992. An augmented lagrangian treatment of contact problems involving friction. Comp. Struct. 42, 97-116.

[35] Taylor, R. L., Papadopoulios, P., 1991. On a patch test for contact problems in two dimensions. Nonlinear Comp. Mech., 690-702.

[36] Tur, M., Fuenmayor, F., Wriggers, P., 2009. A mortar-based frictional contact formulation for large deformations using lagrange multipliers. Comp. Meth. Appl. Mech. Eng. 198, 2860-2873.

[37] Wriggers, P., 2006. Computational Contact Mechanics (2nd edn). Springer, Berlin.

[38] Wriggers, P., Zavarise, G., 2008. A formulation for frictionless contact problems using a weak form introduced by Nitsche. Comp. Mech. 41. 